\title{
Improved Prediction for Methane Activation Mechanism on Rutile Metal Oxides by a Machine Learning Model with Geometrical Descriptors - the Supporting Information
}

\author{
Jiayan Xu, ${ }^{a, b}$ Xiao-Ming Cao, ${ }^{a,}{ }^{*}$ P. Hu ${ }^{a, b}$
}

a Key Laboratory for Advanced Materials, Centre for Computational Chemistry and Research Institute of Industrial Catalysis, School of Chemistry and Molecular Engineering, East China University of Science and Technology, 130 Meilong Road, Shanghai, P. R. China 200237

b School of Chemistry and Chemical Engineering, The Queen's University of Belfast, Belfast BT9 5AG, U.K.

Corresponding Author

*E-mail address: xmcao@ecust.edu.cn

\section{CONTENTS}

S1. Energetic and Geometrical Data

S2. Concepts of LASSO and Percentile-LASSO

S3. Comparasion of Different Regression Methods

S4. References 


\section{Energetic and Geometrical Data}

Table S1 gives adsoprtion energies and reaction barriers (two mechanisms) of 100 rutile-type metal oxides with the zero point energy correction. $E_{\mathrm{CH}_{3}}$ on Ru-dopped $\mathrm{CrO}_{2}$ (110) is not converged due to the magenetic electronic structure. Some of reaction barriers are not calculated because of the limination of the transition state search method.

Table S2 gives input energetic and geomrtrcial descriptors, which includes 5 subtables (from (1) to (5)). The unit for energy is $\mathrm{eV}$, for distance is $\AA$ and for angle and dihedral is rad. $E_{H^{*}}$ is the adsorption energy of $\mathrm{H}$ at the inclined position. $\mathrm{E}_{\mathrm{CH}_{3}^{*}}$ is the adsorption energy of $\mathrm{CH}_{3}$ at the vertical position. There are 65 samples of the surface-stabilized mechanism and 35 samples of the radical mechanism in total. Reaction barriers of both two mechanims are calculated on 22 sufaces.

Table S1 Adsorption Energies and Reaction Barriers (eV)

\begin{tabular}{ccccccc}
\hline Structure $(\mathrm{Base} / \mathrm{Dopant})$ & $E_{H^{o t}}$ & $E_{H^{\text {in }}}$ & $E_{\mathrm{CH}_{3}^{v}}$ & $E_{C H_{3}}$ & $E_{S S}$ & $E_{r a}$ \\
\hline $\mathrm{CrO}_{2} / \mathrm{Ge}$ & -3.27 & -3.30 & -1.26 & -1.26 & 0.90 & 0.75 \\
$\mathrm{CrO}_{2} / \mathrm{Ir}$ & -3.22 & -3.21 & -2.59 & -2.64 & n.c. & 0.76 \\
$\mathrm{CrO}_{2} / \mathrm{Mn}$ & -3.29 & -3.32 & -1.32 & -1.29 & 0.61 & 0.73 \\
$\mathrm{CrO}_{2} / \mathrm{Mo}$ & -3.24 & -3.24 & -1.12 & -1.12 & n.c. & 0.78 \\
$\mathrm{CrO}_{2} / \mathrm{Os}$ & -3.20 & -3.19 & -2.21 & -2.23 & 0.31 & n.c. \\
$\mathrm{CrO}_{2} / \mathrm{Rh}$ & -3.22 & -3.22 & -2.14 & -2.18 & n.c. & n.c. \\
$\mathrm{CrO}_{2} / \mathrm{Ru}$ & -3.22 & -3.21 & -1.82 & n.c. & n.c. & n.c. \\
$\mathrm{CrO}_{2} / \mathrm{Ti}$ & -3.27 & -3.28 & -0.44 & -0.44 & 0.89 & 0.77 \\
$\mathrm{CrO}_{2} / \mathrm{V}$ & -3.26 & -3.29 & -0.44 & -0.45 & 0.91 & 0.75 \\
$\mathrm{GeO}_{2} / \mathrm{Cr}$ & -3.90 & -3.92 & -0.69 & -0.69 & n.c. & n.c. \\
$\mathrm{GeO}_{2} / \mathrm{Ir}$ & -3.50 & -3.53 & -2.80 & -2.80 & -0.62 & n.c. \\
$\mathrm{GeO}_{2} / \mathrm{Mn}$ & -3.95 & -3.98 & -0.91 & -0.91 & n.c. & n.c. \\
$\mathrm{GeO}_{2} / \mathrm{Mo}$ & -2.61 & -2.64 & -2.03 & -2.03 & n.c. & n.c. \\
$\mathrm{GeO}_{2} / \mathrm{Os}$ & -2.88 & -2.89 & -2.46 & -2.46 & n.c. & n.c. \\
$\mathrm{GeO}_{2} / \mathrm{Rh}$ & -3.92 & -3.95 & -2.11 & -2.10 & n.c. & n.c. \\
$\mathrm{GeO}_{2} / \mathrm{Ru}$ & -3.26 & -3.29 & -1.77 & -1.77 & n.c. & n.c. \\
$\mathrm{GeO}_{2} / \mathrm{Ti}$ & -2.92 & -2.96 & -0.55 & -0.55 & 0.46 & 1.01 \\
$\mathrm{GeO}_{2} / \mathrm{V}$ & -3.07 & -3.10 & -0.57 & -0.57 & 0.47 & 0.93 \\
$\mathrm{IrO}_{2} / \mathrm{Cr}$ & -3.23 & -3.31 & -1.01 & -1.01 & 0.39 & n.c. \\
$\mathrm{IrO}_{2} / \mathrm{Ge}$ & -3.15 & -3.24 & -1.89 & -1.89 & n.c. & 0.74 \\
$\mathrm{IrO}_{2} / \mathrm{Mn}$ & -3.29 & -3.38 & -1.28 & -1.28 & 0.51 & 0.60 \\
$\mathrm{IrO}_{2} / \mathrm{Mo}$ & -3.17 & -3.23 & -1.62 & -1.61 & n.c. & n.c. \\
$\mathrm{IrO}_{2} / \mathrm{Os}$ & -3.15 & -3.21 & -2.51 & -2.51 & -0.42 & n.c. \\
$\mathrm{IrO}_{2} / \mathrm{Rh}$ & -3.24 & -3.30 & -2.28 & -2.28 & n.c. & n.c. \\
$\mathrm{IrO}_{2} / \mathrm{Ru}$ & -3.19 & -3.25 & -1.97 & -1.97 & -0.17 & n.c. \\
$\mathrm{IrO}_{2} / \mathrm{Ti}$ & -3.23 & -3.30 & -0.69 & -0.69 & 0.34 & n.c. \\
$\mathrm{IrO}_{2} / \mathrm{V}$ & -3.24 & -3.32 & -0.93 & -0.92 & 0.32 & n.c. \\
$\mathrm{MnO}_{2} / \mathrm{Cr}$ & -4.07 & -4.53 & -1.45 & -1.44 & -0.19 & n.c. \\
\hline & & & & & &
\end{tabular}




\begin{tabular}{|c|c|c|c|c|c|c|}
\hline $\mathrm{MnO}_{2} / \mathrm{Ge}$ & -3.55 & -3.67 & -1.04 & -1.04 & n.c. & 0.44 \\
\hline $\mathrm{MnO}_{2} / \mathrm{Ir}$ & -3.51 & -3.56 & -2.39 & -2.38 & n.c. & n.c. \\
\hline $\mathrm{MnO}_{2} / \mathrm{Mo}$ & -3.91 & -3.80 & -0.92 & -0.91 & 0.43 & 0.78 \\
\hline $\mathrm{MnO}_{2} / \mathrm{Os}$ & -3.53 & -3.59 & -1.97 & -1.97 & n.c. & n.c. \\
\hline $\mathrm{MnO}_{2} / \mathrm{Rh}$ & -3.61 & -3.68 & -1.99 & -1.98 & n.c. & n.c. \\
\hline $\mathrm{MnO}_{2} / \mathrm{Ru}$ & -3.59 & -3.65 & -1.63 & -1.63 & n.c. & n.c. \\
\hline $\mathrm{MnO}_{2} / \mathrm{Ti}$ & -3.63 & -4.51 & -1.01 & -1.02 & n.c. & -0.12 \\
\hline $\mathrm{MnO}_{2} / \mathrm{V}$ & -3.07 & -2.85 & -0.36 & -0.36 & n.c. & n.c. \\
\hline $\mathrm{MoO}_{2} / \mathrm{Cr}$ & -2.80 & -2.86 & -1.46 & -1.54 & n.c. & n.c. \\
\hline $\mathrm{MoO}_{2} / \mathrm{Ge}$ & -2.79 & -2.88 & -1.82 & -1.82 & n.c. & 1.15 \\
\hline $\mathrm{MoO}_{2} / \mathrm{Ir}$ & -3.18 & -3.26 & -3.26 & -3.25 & -0.01 & n.c. \\
\hline $\mathrm{MoO}_{2} / \mathrm{Mn}$ & -2.78 & -2.86 & -1.70 & -1.70 & n.c. & 1.21 \\
\hline $\mathrm{MoO}_{2} / \mathrm{Os}$ & -3.30 & -3.30 & -3.31 & -3.28 & -0.09 & n.c. \\
\hline $\mathrm{MoO}_{2} / \mathrm{Rh}$ & -3.07 & -3.13 & -2.66 & -2.65 & 0.42 & n.c. \\
\hline $\mathrm{MoO}_{2} / \mathrm{Ru}$ & -3.15 & -3.21 & -2.81 & -2.81 & 0.18 & n.c. \\
\hline $\mathrm{MoO}_{2} / \mathrm{Ti}$ & -3.08 & -3.07 & -0.88 & -0.89 & 0.87 & 1.04 \\
\hline $\mathrm{MoO}_{2} / \mathrm{V}$ & -2.82 & -2.93 & -1.32 & -1.30 & 0.69 & 1.11 \\
\hline $\mathrm{OsO}_{2} / \mathrm{Cr}$ & -2.70 & -2.69 & -1.11 & -1.11 & 0.65 & n.c. \\
\hline $\mathrm{OsO}_{2} / \mathrm{Ge}$ & -2.70 & -2.69 & -1.87 & -1.87 & n.c. & n.c. \\
\hline $\mathrm{OsO}_{2} / \mathrm{Ir}$ & -2.69 & -2.68 & -2.68 & -2.68 & -0.19 & n.c. \\
\hline $\mathrm{OsO}_{2} / \mathrm{Mn}$ & -2.73 & -2.72 & -1.31 & -1.31 & 0.71 & n.c. \\
\hline $\mathrm{OsO}_{2} / \mathrm{Mo}$ & -2.60 & -2.59 & -1.58 & -1.58 & 0.25 & n.c. \\
\hline $\mathrm{OsO}_{2} / \mathrm{Rh}$ & -2.69 & -2.68 & -2.12 & -2.11 & n.c. & n.c. \\
\hline $\mathrm{OsO}_{2} / \mathrm{Ru}$ & -2.66 & -2.65 & -1.91 & -1.91 & 0.15 & n.c. \\
\hline $\mathrm{OsO}_{2} / \mathrm{Ti}$ & -2.70 & -2.69 & -0.85 & -0.83 & 0.69 & n.c. \\
\hline $\mathrm{OsO}_{2} / \mathrm{V}$ & -2.68 & -2.65 & -0.96 & -0.96 & 0.65 & n.c. \\
\hline $\mathrm{RhO}_{2} / \mathrm{Cr}$ & -3.67 & -3.81 & -0.65 & -0.65 & 0.45 & n.c. \\
\hline $\mathrm{RhO}_{2} / \mathrm{Ge}$ & -3.61 & -3.76 & -1.22 & -1.22 & 0.51 & 0.37 \\
\hline $\mathrm{RhO}_{2} / \mathrm{Ir}$ & -3.65 & -3.75 & -2.43 & -2.43 & -0.39 & n.c. \\
\hline $\mathrm{RhO}_{2} / \mathrm{Mn}$ & -3.75 & -3.88 & -1.09 & -1.09 & 0.37 & n.c. \\
\hline $\mathrm{RhO}_{2} / \mathrm{Mo}$ & -3.60 & -3.72 & -1.16 & -1.15 & 0.04 & 0.35 \\
\hline $\mathrm{RhO}_{2} / \mathrm{Os}$ & -3.59 & -3.68 & -2.15 & -2.15 & n.c. & n.c. \\
\hline $\mathrm{RhO}_{2} / \mathrm{Ru}$ & -3.65 & -3.75 & -1.68 & -1.69 & n.c. & 0.32 \\
\hline $\mathrm{RhO}_{2} / \mathrm{Ti}$ & -3.73 & -3.86 & -0.58 & -0.57 & 0.27 & 0.25 \\
\hline $\mathrm{RhO}_{2} / \mathrm{V}$ & -3.64 & -3.78 & -0.55 & -0.55 & 0.39 & 0.34 \\
\hline $\mathrm{RuO}_{2} / \mathrm{Cr}$ & -3.09 & -3.16 & -0.77 & -0.77 & 0.66 & n.c. \\
\hline $\mathrm{RuO}_{2} / \mathrm{Ge}$ & -3.09 & -3.15 & -1.55 & -1.55 & n.c. & 0.87 \\
\hline $\mathrm{RuO}_{2} / \mathrm{Ir}$ & -3.06 & -3.09 & -2.47 & -2.47 & -0.20 & n.c. \\
\hline $\mathrm{RuO}_{2} / \mathrm{Mn}$ & -3.11 & -3.19 & -1.16 & -1.16 & 0.59 & 0.71 \\
\hline $\mathrm{RuO}_{2} / \mathrm{Mo}$ & -3.01 & -3.06 & -1.24 & -1.25 & 0.28 & n.c. \\
\hline
\end{tabular}




\begin{tabular}{|c|c|c|c|c|c|c|}
\hline $\mathrm{RuO}_{2} / \mathrm{Os}$ & -3.02 & -3.06 & -2.23 & -2.23 & -0.12 & n.c. \\
\hline $\mathrm{RuO}_{2} / \mathrm{Rh}$ & -3.07 & -3.10 & -1.93 & -1.93 & 0.09 & n.c. \\
\hline $\mathrm{RuO}_{2} / \mathrm{Ti}$ & -3.09 & -3.16 & -0.47 & -0.48 & 0.67 & 0.83 \\
\hline $\mathrm{RuO}_{2} / \mathrm{V}$ & -3.08 & -3.16 & -0.65 & -0.69 & 0.64 & 0.83 \\
\hline $\mathrm{TiO}_{2} / \mathrm{Cr}$ & -3.30 & -3.32 & -0.54 & -0.54 & 0.75 & n.c. \\
\hline $\mathrm{TiO}_{2} / \mathrm{Ge}$ & -2.57 & -2.57 & -0.49 & -0.49 & 0.82 & n.c. \\
\hline $\mathrm{TiO}_{2} / \mathrm{Ir}$ & -3.34 & -3.34 & -2.33 & -2.32 & -0.32 & n.c. \\
\hline $\mathrm{TiO}_{2} / \mathrm{Mn}$ & -3.59 & -3.60 & -0.77 & -0.77 & 0.46 & n.c. \\
\hline $\mathrm{TiO}_{2} / \mathrm{Mo}$ & -2.54 & -2.53 & -1.28 & -1.28 & 0.55 & n.c. \\
\hline $\mathrm{TiO}_{2} / \mathrm{Os}$ & -2.76 & -2.75 & -2.42 & -2.42 & n.c. & n.c. \\
\hline $\mathrm{TiO}_{2} / \mathrm{Rh}$ & -3.70 & -3.70 & -1.77 & -1.77 & -0.09 & 0.53 \\
\hline $\mathrm{TiO}_{2} / \mathrm{Ru}$ & -3.02 & -3.02 & -1.86 & -1.86 & 0.15 & n.c. \\
\hline $\mathrm{TiO}_{2} / \mathrm{V}$ & -2.69 & -2.70 & -0.80 & -0.72 & 0.99 & n.c. \\
\hline $\mathrm{VO}_{2} / \mathrm{Cr}$ & -2.48 & -2.50 & -1.08 & -1.08 & 0.98 & n.c. \\
\hline $\mathrm{VO}_{2} / \mathrm{Ge}$ & -2.46 & -2.54 & -1.81 & -1.80 & n.c. & 1.40 \\
\hline $\mathrm{VO}_{2} / \mathrm{Ir}$ & -2.46 & -2.49 & -2.69 & -2.68 & n.c. & n.c. \\
\hline $\mathrm{VO}_{2} / \mathrm{Mn}$ & -2.45 & -2.49 & -1.38 & -1.38 & 1.06 & n.c. \\
\hline $\mathrm{VO}_{2} / \mathrm{Mo}$ & -2.44 & -2.59 & -1.10 & -1.10 & 0.98 & n.c. \\
\hline $\mathrm{VO}_{2} / \mathrm{Os}$ & -2.46 & -2.50 & -2.56 & -2.56 & 0.26 & n.c. \\
\hline $\mathrm{VO}_{2} / \mathrm{Rh}$ & -2.56 & -2.56 & -2.30 & -2.29 & n.c. & n.c. \\
\hline $\mathrm{VO}_{2} / \mathrm{Ru}$ & -2.49 & -2.52 & -2.13 & -2.12 & 0.48 & n.c. \\
\hline $\mathrm{VO}_{2} / \mathrm{Ti}$ & -2.48 & -2.62 & -0.76 & -0.75 & 1.16 & 1.48 \\
\hline $\mathrm{CrO}_{2}$ & -3.26 & -3.28 & -0.86 & -0.86 & 0.68 & 0.77 \\
\hline $\mathrm{GeO}_{2}$ & -2.72 & -2.75 & -0.59 & -0.59 & n.c. & 1.23 \\
\hline $\mathrm{IrO}_{2}$ & -3.21 & -3.26 & -2.77 & -2.78 & -0.46 & n.c. \\
\hline $\mathrm{MnO}_{2}$ & -3.64 & -3.74 & -1.07 & -1.07 & n.c. & 0.39 \\
\hline $\mathrm{MoO}_{2}$ & -2.82 & -2.91 & -1.94 & -1.95 & 0.11 & n.c. \\
\hline $\mathrm{OsO}_{2}$ & -2.64 & -2.63 & -2.49 & -2.49 & -0.16 & n.c. \\
\hline $\mathrm{RhO}_{2}$ & -3.71 & -3.81 & -1.98 & -1.98 & n.c. & 0.28 \\
\hline $\mathrm{RuO}_{2}$ & -3.05 & -3.09 & -1.70 & -1.70 & 0.13 & n.c. \\
\hline $\mathrm{TiO}_{2}$ & -2.49 & -2.47 & -0.46 & -0.47 & 0.95 & 1.59 \\
\hline $\mathrm{VO}_{2}$ & -2.48 & -2.55 & -0.78 & -0.70 & 1.27 & 1.32 \\
\hline & & & & & \multicolumn{2}{|c|}{ (n.c.: not calculated) } \\
\hline
\end{tabular}


Table S2 Input Energetic and Geometrical Descriptors (1)

\begin{tabular}{|c|c|c|c|c|c|c|}
\hline $\begin{array}{c}\text { Structure (Base / } \\
\text { Dopant) }\end{array}$ & $\overline{E_{H^{*}}}$ & $\overline{E_{C H_{3}^{*}}}$ & $D_{O_{b r}}^{\text {suf }}-M_{c u s}$ & $D_{O_{b r}-M_{c u s}}^{H^{*}}$ & $D_{O_{b r}-H}^{H^{*}}$ & $D_{M_{\text {cus }}-H}^{H^{*}}$ \\
\hline $\mathrm{CrO}_{2} / \mathrm{Ge}$ & -3.30 & -1.26 & 3.414 & 3.541 & 0.973 & 3.250 \\
\hline $\mathrm{CrO}_{2} / \mathrm{Ir}$ & -3.21 & -2.59 & 3.348 & 3.539 & 0.972 & 3.503 \\
\hline $\mathrm{CrO}_{2} / \mathrm{Mn}$ & -3.31 & -1.32 & 3.368 & 3.494 & 0.974 & 3.209 \\
\hline $\mathrm{CrO}_{2} / \mathrm{Mo}$ & -3.24 & -1.12 & 3.420 & 3.604 & 0.972 & 3.451 \\
\hline $\mathrm{CrO}_{2} / \mathrm{Os}$ & -3.19 & -2.21 & 3.367 & 3.537 & 0.972 & 3.454 \\
\hline $\mathrm{CrO}_{2} / \mathrm{Ti}$ & -3.28 & -0.44 & 3.397 & 3.554 & 0.973 & 3.321 \\
\hline $\mathrm{CrO}_{2} / \mathrm{V}$ & -3.29 & -0.44 & 3.425 & 3.557 & 0.974 & 3.267 \\
\hline $\mathrm{GeO}_{2} / \mathrm{Ir}$ & -3.53 & -2.80 & 3.417 & 3.505 & 0.974 & 3.237 \\
\hline $\mathrm{GeO}_{2} / \mathrm{Ti}$ & -2.96 & -0.55 & 3.460 & 3.585 & 0.976 & 3.318 \\
\hline $\mathrm{GeO}_{2} / \mathrm{V}$ & -3.09 & -0.57 & 3.487 & 3.570 & 0.976 & 3.289 \\
\hline $\mathrm{IrO}_{2} / \mathrm{Cr}$ & -3.31 & -1.01 & 3.470 & 3.594 & 0.977 & 3.284 \\
\hline $\mathrm{IrO}_{2} / \mathrm{Ge}$ & -3.24 & -1.89 & 3.465 & 3.596 & 0.977 & 3.275 \\
\hline $\mathrm{IrO}_{2} / \mathrm{Mn}$ & -3.38 & -1.28 & 3.449 & 3.558 & 0.978 & 3.187 \\
\hline $\mathrm{IrO}_{2} / \mathrm{Os}$ & -3.21 & -2.51 & 3.459 & 3.611 & 0.976 & 3.403 \\
\hline $\mathrm{IrO}_{2} / \mathrm{Ru}$ & -3.24 & -1.97 & 3.454 & 3.572 & 0.976 & 3.304 \\
\hline $\mathrm{IrO}_{2} / \mathrm{Ti}$ & -3.30 & -0.69 & 3.466 & 3.620 & 0.976 & 3.318 \\
\hline $\mathrm{IrO}_{2} / \mathrm{V}$ & -3.31 & -0.93 & 3.482 & 3.615 & 0.977 & 3.309 \\
\hline $\mathrm{MnO}_{2} / \mathrm{Cr}$ & -4.52 & -1.45 & 3.411 & 3.545 & 0.977 & 3.168 \\
\hline $\mathrm{MnO}_{2} / \mathrm{Ge}$ & -3.67 & -1.04 & 3.411 & 3.542 & 0.977 & 3.140 \\
\hline $\mathrm{MnO}_{2} / \mathrm{Mo}$ & -3.80 & -0.92 & 3.447 & 3.605 & 0.975 & 3.333 \\
\hline $\mathrm{MnO}_{2} / \mathrm{Ti}$ & -4.50 & -1.01 & 3.408 & 3.567 & 0.977 & 3.165 \\
\hline $\mathrm{MoO}_{2} / \mathrm{Ge}$ & -2.88 & -1.82 & 3.974 & 4.112 & 0.975 & 3.833 \\
\hline $\mathrm{MoO}_{2} / \mathrm{Ir}$ & -3.26 & -3.26 & 3.843 & 3.959 & 0.975 & 3.658 \\
\hline $\mathrm{MoO}_{2} / \mathrm{Mn}$ & -2.85 & -1.70 & 3.847 & 4.019 & 0.975 & 3.734 \\
\hline $\mathrm{MoO}_{2} / \mathrm{Os}$ & -3.29 & -3.31 & 3.921 & 4.067 & 0.974 & 3.827 \\
\hline $\mathrm{MoO}_{2} / \mathrm{Rh}$ & -3.13 & -2.66 & 3.845 & 3.936 & 0.975 & 3.621 \\
\hline $\mathrm{MoO}_{2} / \mathrm{Ru}$ & -3.21 & -2.81 & 3.908 & 4.060 & 0.974 & 3.836 \\
\hline $\mathrm{MoO}_{2} / \mathrm{Ti}$ & -3.07 & -0.88 & 3.873 & 3.942 & 0.969 & 4.132 \\
\hline $\mathrm{MoO}_{2} / \mathrm{V}$ & -2.92 & -1.32 & 3.927 & 4.144 & 0.975 & 3.960 \\
\hline $\mathrm{OsO}_{2} / \mathrm{Cr}$ & -2.69 & -1.11 & 3.439 & 3.606 & 0.973 & 3.429 \\
\hline $\mathrm{OsO}_{2} / \mathrm{Ir}$ & -2.67 & -2.68 & 3.401 & 3.531 & 0.973 & 3.383 \\
\hline $\mathrm{OsO}_{2} / \mathrm{Mn}$ & -2.72 & -1.31 & 3.430 & 3.573 & 0.974 & 3.385 \\
\hline $\mathrm{OsO}_{2} / \mathrm{Mo}$ & -2.59 & -1.58 & 3.445 & 3.629 & 0.973 & 3.496 \\
\hline $\mathrm{OsO}_{2} / \mathrm{Ru}$ & -2.65 & -1.91 & 3.423 & 3.573 & 0.975 & 3.383 \\
\hline $\mathrm{OsO}_{2} / \mathrm{Ti}$ & -2.69 & -0.85 & 3.461 & 3.622 & 0.973 & 3.477 \\
\hline $\mathrm{OsO}_{2} / \mathrm{V}$ & -2.65 & -0.96 & 3.467 & 3.622 & 0.974 & 3.405 \\
\hline $\mathrm{RhO}_{2} / \mathrm{Cr}$ & -3.81 & -0.65 & 3.481 & 3.639 & 0.977 & 3.293 \\
\hline
\end{tabular}




\begin{tabular}{|c|c|c|c|c|c|c|}
\hline $\mathrm{RhO}_{2} / \mathrm{Ge}$ & -3.76 & -1.22 & 3.461 & 3.603 & 0.979 & 3.209 \\
\hline $\mathrm{RhO}_{2} / \mathrm{Ir}$ & -3.75 & -2.43 & 3.444 & 3.624 & 0.977 & 3.362 \\
\hline $\mathrm{RhO}_{2} / \mathrm{Mn}$ & -3.88 & -1.09 & 3.438 & 3.575 & 0.978 & 3.150 \\
\hline $\mathrm{RhO}_{2} / \mathrm{Mo}$ & -3.71 & -1.16 & 3.479 & 3.710 & 0.976 & 3.491 \\
\hline $\mathrm{RhO}_{2} / \mathrm{Ru}$ & -3.75 & -1.68 & 3.466 & 3.653 & 0.976 & 3.383 \\
\hline $\mathrm{RhO}_{2} / \mathrm{Ti}$ & -3.85 & -0.58 & 3.464 & 3.635 & 0.977 & 3.270 \\
\hline $\mathrm{RhO}_{2} / \mathrm{V}$ & -3.78 & -0.55 & 3.483 & 3.630 & 0.977 & 3.251 \\
\hline $\mathrm{RuO}_{2} / \mathrm{Cr}$ & -3.16 & -0.77 & 3.464 & 3.628 & 0.975 & 3.301 \\
\hline $\mathrm{RuO}_{2} / \mathrm{Ge}$ & -3.15 & -1.55 & 3.458 & 3.621 & 0.975 & 3.295 \\
\hline $\mathrm{RuO}_{2} / \mathrm{Ir}$ & -3.09 & -2.47 & 3.414 & 3.581 & 0.975 & 3.293 \\
\hline $\mathrm{RuO}_{2} / \mathrm{Mn}$ & -3.19 & -1.16 & 3.437 & 3.572 & 0.976 & 3.183 \\
\hline $\mathrm{RuO}_{2} / \mathrm{Mo}$ & -3.06 & -1.24 & 3.476 & 3.679 & 0.975 & 3.420 \\
\hline $\mathrm{RuO}_{2} / \mathrm{Os}$ & -3.06 & -2.23 & 3.443 & 3.636 & 0.975 & 3.418 \\
\hline $\mathrm{RuO}_{2} / \mathrm{Rh}$ & -3.10 & -1.93 & 3.407 & 3.568 & 0.975 & 3.271 \\
\hline $\mathrm{RuO}_{2} / \mathrm{Ti}$ & -3.16 & -0.47 & 3.467 & 3.648 & 0.976 & 3.334 \\
\hline $\mathrm{RuO}_{2} / \mathrm{V}$ & -3.16 & -0.65 & 3.482 & 3.637 & 0.976 & 3.295 \\
\hline $\mathrm{TiO}_{2} / \mathrm{Cr}$ & -3.31 & -0.54 & 3.599 & 3.618 & 0.973 & 3.284 \\
\hline $\mathrm{TiO}_{2} / \mathrm{Ge}$ & -2.57 & -0.49 & 3.590 & 3.615 & 0.974 & 3.309 \\
\hline $\mathrm{TiO}_{2} / \mathrm{Ir}$ & -3.34 & -2.33 & 3.552 & 3.585 & 0.972 & 3.314 \\
\hline $\mathrm{TiO}_{2} / \mathrm{Mn}$ & -3.59 & -0.77 & 3.576 & 3.563 & 0.973 & 3.244 \\
\hline $\mathrm{TiO}_{2} / \mathrm{Mo}$ & -2.53 & -1.28 & 3.586 & 3.641 & 0.971 & 3.470 \\
\hline $\mathrm{TiO}_{2} / \mathrm{Rh}$ & -3.70 & -1.77 & 3.555 & 3.591 & 0.972 & 3.314 \\
\hline $\mathrm{TiO}_{2} / \mathrm{Ru}$ & -3.02 & -1.86 & 3.563 & 3.604 & 0.973 & 3.296 \\
\hline $\mathrm{TiO}_{2} / \mathrm{V}$ & -2.70 & -0.80 & 3.604 & 3.627 & 0.974 & 3.286 \\
\hline $\mathrm{VO}_{2} / \mathrm{Cr}$ & -2.50 & -1.08 & 3.517 & 3.572 & 0.974 & 3.222 \\
\hline $\mathrm{VO}_{2} / \mathrm{Ge}$ & -2.53 & -1.81 & 3.521 & 3.580 & 0.974 & 3.223 \\
\hline $\mathrm{VO}_{2} / \mathrm{Mn}$ & -2.49 & -1.38 & 3.470 & 3.547 & 0.975 & 3.190 \\
\hline $\mathrm{VO}_{2} / \mathrm{Mo}$ & -2.59 & -1.10 & 3.552 & 3.667 & 0.974 & 3.400 \\
\hline $\mathrm{VO}_{2} / \mathrm{Os}$ & -2.50 & -2.56 & 3.504 & 3.644 & 0.973 & 3.434 \\
\hline $\mathrm{VO}_{2} / \mathrm{Ru}$ & -2.52 & -2.13 & 3.499 & 3.599 & 0.974 & 3.357 \\
\hline $\mathrm{VO}_{2} / \mathrm{Ti}$ & -2.62 & -0.76 & 3.524 & 3.636 & 0.975 & 3.336 \\
\hline $\mathrm{CrO}_{2}$ & -3.28 & -0.86 & 3.414 & 3.548 & 0.973 & 3.306 \\
\hline $\mathrm{GeO}_{2}$ & -2.75 & -0.59 & 3.467 & 3.582 & 0.976 & 3.324 \\
\hline $\mathrm{IrO}_{2}$ & -3.26 & -2.77 & 3.443 & 3.532 & 0.977 & 3.210 \\
\hline $\mathrm{MnO}_{2}$ & -3.74 & -1.07 & 3.390 & 3.505 & 0.977 & 3.109 \\
\hline $\mathrm{MoO}_{2}$ & -2.91 & -1.94 & 3.897 & 4.103 & 0.974 & 3.873 \\
\hline $\mathrm{OsO}_{2}$ & -2.63 & -2.49 & 3.424 & 3.566 & 0.974 & 3.345 \\
\hline $\mathrm{RhO}_{2}$ & -3.81 & -1.98 & 3.440 & 3.563 & 0.978 & 3.188 \\
\hline $\mathrm{RuO}_{2}$ & -3.09 & -1.70 & 3.436 & 3.621 & 0.974 & 3.359 \\
\hline $\mathrm{TiO}_{2}$ & -2.47 & -0.46 & 3.584 & 3.652 & 0.972 & 3.472 \\
\hline
\end{tabular}




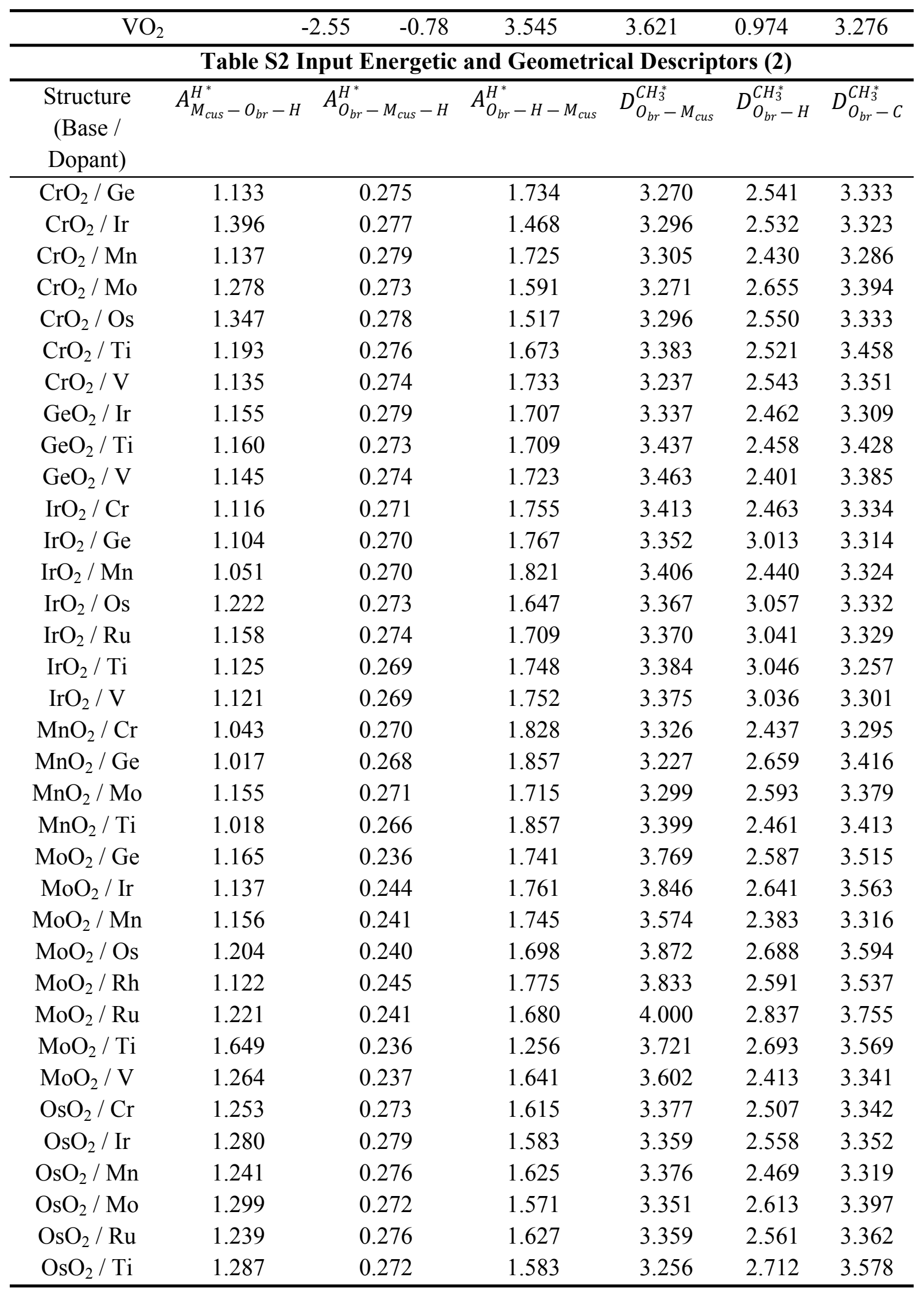




\begin{tabular}{|c|c|c|c|c|c|c|}
\hline $\mathrm{OsO}_{2} / \mathrm{V}$ & 1.212 & 0.271 & 1.658 & 3.360 & 2.540 & 3.359 \\
\hline $\mathrm{RhO}_{2} / \mathrm{Cr}$ & 1.080 & 0.265 & 1.797 & 3.425 & 2.416 & 3.316 \\
\hline $\mathrm{RhO}_{2} / \mathrm{Ge}$ & 1.028 & 0.264 & 1.849 & 3.372 & 2.475 & 3.329 \\
\hline $\mathrm{RhO}_{2} / \mathrm{Ir}$ & 1.167 & 0.270 & 1.704 & 3.391 & 2.498 & 3.329 \\
\hline $\mathrm{RhO}_{2} / \mathrm{Mn}$ & 0.993 & 0.263 & 1.885 & 3.398 & 2.427 & 3.315 \\
\hline $\mathrm{RhO}_{2} / \mathrm{Mo}$ & 1.213 & 0.265 & 1.663 & 3.361 & 2.590 & 3.398 \\
\hline $\mathrm{RhO}_{2} / \mathrm{Ru}$ & 1.159 & 0.268 & 1.715 & 3.381 & 2.507 & 3.340 \\
\hline $\mathrm{RhO}_{2} / \mathrm{Ti}$ & 1.060 & 0.264 & 1.818 & 3.450 & 2.463 & 3.428 \\
\hline $\mathrm{RhO}_{2} / \mathrm{V}$ & 1.045 & 0.263 & 1.834 & 3.470 & 2.420 & 3.371 \\
\hline $\mathrm{RuO}_{2} / \mathrm{Cr}$ & 1.099 & 0.266 & 1.776 & 3.399 & 2.464 & 3.326 \\
\hline $\mathrm{RuO}_{2} / \mathrm{Ge}$ & 1.099 & 0.267 & 1.775 & 3.360 & 2.501 & 3.336 \\
\hline $\mathrm{RuO}_{2} / \mathrm{Ir}$ & 1.138 & 0.272 & 1.732 & 3.361 & 2.555 & 3.352 \\
\hline $\mathrm{RuO}_{2} / \mathrm{Mn}$ & 1.032 & 0.266 & 1.843 & 3.375 & 2.453 & 3.312 \\
\hline $\mathrm{RuO}_{2} / \mathrm{Mo}$ & 1.171 & 0.266 & 1.705 & 3.351 & 2.603 & 3.404 \\
\hline $\mathrm{RuO}_{2} / \mathrm{Os}$ & 1.212 & 0.270 & 1.659 & 3.364 & 2.569 & 3.360 \\
\hline $\mathrm{RuO}_{2} / \mathrm{Rh}$ & 1.128 & 0.273 & 1.741 & 3.366 & 2.511 & 3.340 \\
\hline $\mathrm{RuO}_{2} / \mathrm{Ti}$ & 1.114 & 0.266 & 1.762 & 3.449 & 2.480 & 3.432 \\
\hline $\mathrm{RuO}_{2} / \mathrm{V}$ & 1.084 & 0.265 & 1.792 & 3.242 & 2.556 & 3.448 \\
\hline $\mathrm{TiO}_{2} / \mathrm{Cr}$ & 1.090 & 0.266 & 1.786 & 3.579 & 2.448 & 3.436 \\
\hline $\mathrm{TiO}_{2} / \mathrm{Ge}$ & 1.120 & 0.268 & 1.754 & 3.563 & 2.502 & 3.451 \\
\hline $\mathrm{TiO}_{2} / \mathrm{Ir}$ & 1.155 & 0.272 & 1.715 & 3.477 & 2.544 & 3.409 \\
\hline $\mathrm{TiO}_{2} / \mathrm{Mn}$ & 1.104 & 0.271 & 1.767 & 3.552 & 2.420 & 3.407 \\
\hline $\mathrm{TiO}_{2} / \mathrm{Mo}$ & 1.260 & 0.270 & 1.612 & 3.441 & 2.621 & 3.458 \\
\hline $\mathrm{TiO}_{2} / \mathrm{Rh}$ & 1.150 & 0.271 & 1.721 & 3.466 & 2.525 & 3.407 \\
\hline $\mathrm{TiO}_{2} / \mathrm{Ru}$ & 1.118 & 0.269 & 1.755 & 3.450 & 2.573 & 3.425 \\
\hline $\mathrm{TiO}_{2} / \mathrm{V}$ & 1.084 & 0.265 & 1.793 & 3.266 & 2.569 & 3.433 \\
\hline $\mathrm{VO}_{2} / \mathrm{Cr}$ & 1.072 & 0.269 & 1.801 & 3.412 & 2.521 & 3.370 \\
\hline $\mathrm{VO}_{2} / \mathrm{Ge}$ & 1.065 & 0.268 & 1.809 & 3.276 & 2.783 & 3.512 \\
\hline $\mathrm{VO}_{2} / \mathrm{Mn}$ & 1.064 & 0.270 & 1.807 & 3.401 & 2.506 & 3.367 \\
\hline $\mathrm{VO}_{2} / \mathrm{Mo}$ & 1.163 & 0.266 & 1.713 & 3.421 & 2.586 & 3.401 \\
\hline $\mathrm{VO}_{2} / \mathrm{Os}$ & 1.220 & 0.269 & 1.652 & 3.459 & 2.527 & 3.356 \\
\hline $\mathrm{VO}_{2} / \mathrm{Ru}$ & 1.185 & 0.272 & 1.684 & 3.446 & 2.516 & 3.359 \\
\hline $\mathrm{VO}_{2} / \mathrm{Ti}$ & 1.127 & 0.267 & 1.747 & 3.360 & 2.629 & 3.423 \\
\hline $\mathrm{CrO}_{2}$ & 1.185 & 0.276 & 1.681 & 3.297 & 2.469 & 3.294 \\
\hline $\mathrm{GeO}_{2}$ & 1.169 & 0.274 & 1.699 & 3.431 & 2.361 & 3.347 \\
\hline $\mathrm{IrO}_{2}$ & 1.100 & 0.275 & 1.767 & 3.371 & 2.994 & 3.306 \\
\hline $\mathrm{MnO}_{2}$ & 1.022 & 0.271 & 1.848 & 3.312 & 2.438 & 3.300 \\
\hline $\mathrm{MoO}_{2}$ & 1.215 & 0.238 & 1.689 & 3.759 & 2.618 & 3.533 \\
\hline $\mathrm{OsO}_{2}$ & 1.206 & 0.276 & 1.660 & 3.362 & 2.569 & 3.361 \\
\hline $\mathrm{RhO}_{2}$ & 1.046 & 0.269 & 1.827 & 3.391 & 2.448 & 3.311 \\
\hline
\end{tabular}




\begin{tabular}{ccccccc}
\hline $\mathrm{RuO}_{2}$ & 1.166 & 0.270 & 1.706 & 3.369 & 2.526 & 3.349 \\
$\mathrm{TiO}_{2}$ & 1.252 & 0.269 & 1.620 & 3.569 & 2.525 & 3.502 \\
$\mathrm{VO}_{2}$ & 1.079 & 0.265 & 1.797 & 3.407 & 2.513 & 3.359 \\
\hline
\end{tabular}

Table S2 Input Energetic and Geometrical Descriptors (3)

\begin{tabular}{|c|c|c|c|c|c|c|}
\hline $\begin{array}{c}\text { Structure (Base } \\
\text { / Dopant) }\end{array}$ & $D_{M_{\text {cus }}-H}^{C H_{3}^{*}}$ & $D_{M_{c u s}-C}^{C H_{3}^{*}}$ & $D_{H-C}^{C H_{3}^{*}}$ & $A_{M_{c u s}}^{C H_{3}^{*}}-O_{b r}-H$ & $A_{M_{c u s}}^{C H_{3}^{*}}-O_{b r}-C$ & $A_{H-O_{b r}-C}^{C H_{3}^{*}}$ \\
\hline $\mathrm{CrO}_{2} / \mathrm{Ge}$ & 2.551 & 1.999 & 1.095 & 0.876 & 0.615 & 0.261 \\
\hline $\mathrm{CrO}_{2} / \mathrm{Ir}$ & 2.619 & 2.064 & 1.097 & 0.897 & 0.634 & 0.263 \\
\hline $\mathrm{CrO}_{2} / \mathrm{Mn}$ & 2.536 & 2.026 & 1.095 & 0.867 & 0.625 & 0.242 \\
\hline $\mathrm{CrO}_{2} / \mathrm{Mo}$ & 2.686 & 2.122 & 1.100 & 0.919 & 0.647 & 0.272 \\
\hline $\mathrm{CrO}_{2} / \mathrm{Os}$ & 2.644 & 2.087 & 1.099 & 0.905 & 0.640 & 0.265 \\
\hline $\mathrm{CrO}_{2} / \mathrm{Ti}$ & 2.972 & 2.757 & 1.089 & 1.017 & 0.829 & 0.188 \\
\hline $\mathrm{CrO}_{2} / \mathrm{V}$ & 2.577 & 2.071 & 1.099 & 0.894 & 0.639 & 0.256 \\
\hline $\mathrm{GeO}_{2} / \mathrm{Ir}$ & 2.577 & 2.050 & 1.099 & 0.873 & 0.627 & 0.246 \\
\hline $\mathrm{GeO}_{2} / \mathrm{Ti}$ & 2.880 & 2.664 & 1.092 & 0.970 & 0.797 & 0.172 \\
\hline $\mathrm{GeO}_{2} / \mathrm{V}$ & 2.846 & 2.619 & 1.092 & 0.951 & 0.785 & 0.167 \\
\hline $\mathrm{IrO}_{2} / \mathrm{Cr}$ & 2.580 & 2.055 & 1.099 & 0.853 & 0.619 & 0.234 \\
\hline $\mathrm{IrO}_{2} / \mathrm{Ge}$ & 2.551 & 1.988 & 1.093 & 0.818 & 0.606 & 0.334 \\
\hline $\mathrm{IrO}_{2} / \mathrm{Mn}$ & 2.546 & 2.032 & 1.097 & 0.842 & 0.613 & 0.229 \\
\hline $\mathrm{IrO}_{2} / \mathrm{Os}$ & 2.672 & 2.080 & 1.100 & 0.853 & 0.631 & 0.335 \\
\hline $\mathrm{IrO}_{2} / \mathrm{Ru}$ & 2.644 & 2.071 & 1.097 & 0.844 & 0.628 & 0.334 \\
\hline $\mathrm{IrO}_{2} / \mathrm{Ti}$ & 2.759 & 2.147 & 1.093 & 0.881 & 0.657 & 0.342 \\
\hline $\mathrm{IrO}_{2} / \mathrm{V}$ & 2.667 & 2.085 & 1.095 & 0.852 & 0.635 & 0.337 \\
\hline $\mathrm{MnO}_{2} / \mathrm{Cr}$ & 2.572 & 2.064 & 1.096 & 0.875 & 0.634 & 0.241 \\
\hline $\mathrm{MnO}_{2} / \mathrm{Ge}$ & 2.504 & 1.958 & 1.094 & 0.859 & 0.596 & 0.263 \\
\hline $\mathrm{MnO}_{2} / \mathrm{Mo}$ & 2.647 & 2.112 & 1.098 & 0.903 & 0.643 & 0.260 \\
\hline $\mathrm{MnO}_{2} / \mathrm{Ti}$ & 2.896 & 2.666 & 1.090 & 0.987 & 0.804 & 0.183 \\
\hline $\mathrm{MoO}_{2} / \mathrm{Ge}$ & 2.554 & 1.993 & 1.094 & 0.742 & 0.550 & 0.192 \\
\hline $\mathrm{MoO}_{2} / \mathrm{Ir}$ & 2.639 & 2.068 & 1.097 & 0.754 & 0.561 & 0.194 \\
\hline $\mathrm{MoO}_{2} / \mathrm{Mn}$ & 2.545 & 2.008 & 1.095 & 0.791 & 0.587 & 0.204 \\
\hline $\mathrm{MoO}_{2} / \mathrm{Os}$ & 2.679 & 2.096 & 1.099 & 0.763 & 0.564 & 0.201 \\
\hline $\mathrm{MoO}_{2} / \mathrm{Rh}$ & 2.597 & 2.047 & 1.096 & 0.741 & 0.557 & 0.183 \\
\hline $\mathrm{MoO}_{2} / \mathrm{Ru}$ & 2.643 & 2.078 & 1.098 & 0.720 & 0.539 & 0.184 \\
\hline $\mathrm{MoO}_{2} / \mathrm{Ti}$ & 2.672 & 2.107 & 1.100 & 0.800 & 0.585 & 0.215 \\
\hline $\mathrm{MoO}_{2} / \mathrm{V}$ & 2.603 & 2.067 & 1.098 & 0.807 & 0.600 & 0.207 \\
\hline $\mathrm{OsO}_{2} / \mathrm{Cr}$ & 2.596 & 2.052 & 1.098 & 0.867 & 0.621 & 0.247 \\
\hline $\mathrm{OsO}_{2} / \mathrm{Ir}$ & 2.638 & 2.065 & 1.099 & 0.886 & 0.626 & 0.260 \\
\hline $\mathrm{OsO}_{2} / \mathrm{Mn}$ & 2.569 & 2.029 & 1.098 & 0.859 & 0.616 & 0.243 \\
\hline $\mathrm{OsO}_{2} / \mathrm{Mo}$ & 2.697 & 2.145 & 1.102 & 0.907 & 0.647 & 0.261 \\
\hline
\end{tabular}




\begin{tabular}{|c|c|c|c|c|c|c|}
\hline $\mathrm{OsO}_{2} / \mathrm{Ru}$ & 2.639 & 2.080 & 1.099 & 0.886 & 0.629 & 0.257 \\
\hline $\mathrm{OsO}_{2} / \mathrm{Ti}$ & 2.509 & 2.131 & 1.107 & 0.849 & 0.627 & 0.222 \\
\hline $\mathrm{OsO}_{2} / \mathrm{V}$ & 2.631 & 2.088 & 1.101 & 0.884 & 0.632 & 0.252 \\
\hline $\mathrm{RhO}_{2} / \mathrm{Cr}$ & 2.593 & 2.105 & 1.097 & 0.856 & 0.634 & 0.222 \\
\hline $\mathrm{RhO}_{2} / \mathrm{Ge}$ & 2.538 & 2.004 & 1.096 & 0.847 & 0.607 & 0.240 \\
\hline $\mathrm{RhO}_{2} / \mathrm{Ir}$ & 2.630 & 2.080 & 1.097 & 0.878 & 0.629 & 0.249 \\
\hline $\mathrm{RhO}_{2} / \mathrm{Mn}$ & 2.551 & 2.049 & 1.095 & 0.846 & 0.620 & 0.226 \\
\hline $\mathrm{RhO}_{2} / \mathrm{Mo}$ & 2.664 & 2.130 & 1.101 & 0.894 & 0.641 & 0.253 \\
\hline $\mathrm{RhO}_{2} / \mathrm{Ru}$ & 2.633 & 2.096 & 1.097 & 0.882 & 0.634 & 0.247 \\
\hline $\mathrm{RhO}_{2} / \mathrm{Ti}$ & 2.868 & 2.628 & 1.092 & 0.960 & 0.784 & 0.176 \\
\hline $\mathrm{RhO}_{2} / \mathrm{V}$ & 2.794 & 2.473 & 1.090 & 0.926 & 0.739 & 0.187 \\
\hline $\mathrm{RuO}_{2} / \mathrm{Cr}$ & 2.601 & 2.078 & 1.097 & 0.866 & 0.628 & 0.238 \\
\hline $\mathrm{RuO}_{2} / \mathrm{Ge}$ & 2.550 & 2.003 & 1.096 & 0.854 & 0.608 & 0.246 \\
\hline $\mathrm{RuO}_{2} / \mathrm{Ir}$ & 2.641 & 2.074 & 1.098 & 0.887 & 0.628 & 0.259 \\
\hline $\mathrm{RuO}_{2} / \mathrm{Mn}$ & 2.560 & 2.031 & 1.095 & 0.856 & 0.617 & 0.239 \\
\hline $\mathrm{RuO}_{2} / \mathrm{Mo}$ & 2.682 & 2.151 & 1.101 & 0.902 & 0.648 & 0.254 \\
\hline $\mathrm{RuO}_{2} / \mathrm{Os}$ & 2.666 & 2.097 & 1.100 & 0.895 & 0.635 & 0.261 \\
\hline $\mathrm{RuO}_{2} / \mathrm{Rh}$ & 2.601 & 2.058 & 1.097 & 0.872 & 0.624 & 0.249 \\
\hline $\mathrm{RuO}_{2} / \mathrm{Ti}$ & 2.885 & 2.630 & 1.091 & 0.967 & 0.784 & 0.182 \\
\hline $\mathrm{RuO}_{2} / \mathrm{V}$ & 2.500 & 2.121 & 1.102 & 0.861 & 0.642 & 0.219 \\
\hline $\mathrm{TiO}_{2} / \mathrm{Cr}$ & 2.822 & 2.556 & 1.091 & 0.904 & 0.745 & 0.159 \\
\hline $\mathrm{TiO}_{2} / \mathrm{Ge}$ & 2.846 & 2.519 & 1.089 & 0.916 & 0.734 & 0.182 \\
\hline $\mathrm{TiO}_{2} / \mathrm{Ir}$ & 2.599 & 2.070 & 1.098 & 0.840 & 0.610 & 0.230 \\
\hline $\mathrm{TiO}_{2} / \mathrm{Mn}$ & 2.703 & 2.396 & 1.091 & 0.864 & 0.702 & 0.162 \\
\hline $\mathrm{TiO}_{2} / \mathrm{Mo}$ & 2.642 & 2.121 & 1.101 & 0.863 & 0.625 & 0.238 \\
\hline $\mathrm{TiO}_{2} / \mathrm{Rh}$ & 2.566 & 2.061 & 1.095 & 0.831 & 0.609 & 0.222 \\
\hline $\mathrm{TiO}_{2} / \mathrm{Ru}$ & 2.601 & 2.077 & 1.096 & 0.847 & 0.614 & 0.233 \\
\hline $\mathrm{TiO}_{2} / \mathrm{V}$ & 2.526 & 2.095 & 1.101 & 0.865 & 0.634 & 0.230 \\
\hline $\mathrm{VO}_{2} / \mathrm{Cr}$ & 2.576 & 2.042 & 1.095 & 0.849 & 0.611 & 0.238 \\
\hline $\mathrm{VO}_{2} / \mathrm{Ge}$ & 2.521 & 1.959 & 1.095 & 0.844 & 0.582 & 0.262 \\
\hline $\mathrm{VO}_{2} / \mathrm{Mn}$ & 2.540 & 2.015 & 1.095 & 0.838 & 0.605 & 0.234 \\
\hline $\mathrm{VO}_{2} / \mathrm{Mo}$ & 2.704 & 2.161 & 1.100 & 0.894 & 0.644 & 0.250 \\
\hline $\mathrm{VO}_{2} / \mathrm{Os}$ & 2.656 & 2.085 & 1.099 & 0.868 & 0.621 & 0.248 \\
\hline $\mathrm{VO}_{2} / \mathrm{Ru}$ & 2.625 & 2.072 & 1.098 & 0.860 & 0.618 & 0.242 \\
\hline $\mathrm{VO}_{2} / \mathrm{Ti}$ & 2.629 & 2.080 & 1.099 & 0.878 & 0.623 & 0.254 \\
\hline $\mathrm{CrO}_{2}$ & 2.585 & 2.052 & 1.096 & 0.887 & 0.633 & 0.254 \\
\hline $\mathrm{GeO}_{2}$ & 2.733 & 2.475 & 1.093 & 0.915 & 0.747 & 0.168 \\
\hline $\mathrm{IrO}_{2}$ & 2.644 & 2.065 & 1.097 & 0.849 & 0.629 & 0.336 \\
\hline $\mathrm{MnO}_{2}$ & 2.528 & 2.029 & 1.093 & 0.861 & 0.624 & 0.238 \\
\hline $\mathrm{MoO}_{2}$ & 2.685 & 2.140 & 1.104 & 0.796 & 0.593 & 0.203 \\
\hline
\end{tabular}




\begin{tabular}{lllllll}
\hline $\mathrm{OsO}_{2}$ & 2.655 & 2.084 & 1.101 & 0.891 & 0.630 & 0.261 \\
$\mathrm{RhO}_{2}$ & 2.590 & 2.066 & 1.095 & 0.864 & 0.626 & 0.238 \\
$\mathrm{RuO}_{2}$ & 2.630 & 2.088 & 1.097 & 0.882 & 0.632 & 0.250 \\
$\mathrm{TiO}_{2}$ & 2.942 & 2.719 & 1.090 & 0.952 & 0.789 & 0.163 \\
$\mathrm{VO}_{2}$ & 2.598 & 2.059 & 1.097 & 0.860 & 0.618 & 0.241 \\
\hline
\end{tabular}

Table S2 Input Energetic and Geometrical Descriptors (4)

\begin{tabular}{|c|c|c|c|c|c|c|}
\hline $\begin{array}{l}\text { Structur } \\
\text { e (Base / } \\
\text { Dopant) }\end{array}$ & $A_{O_{b r}-M_{c u s}-H}^{C H_{3}^{*}}$ & $A_{O_{b r}-M_{c u s}-C}^{C H_{3}^{*}}$ & $A_{H-M_{c u s}}^{C H_{3}^{*}}-C$ & $A_{O_{b r}-H-M_{c u s}}^{C H_{3}^{*}}$ & $A_{O_{b r}-H-C}^{C H_{3}^{*}}$ & $A_{M_{c u s}}^{C H_{3}^{*}}-H-C$ \\
\hline $\begin{array}{c}\mathrm{CrO}_{2} / \\
\mathrm{Ge}\end{array}$ & 0.871 & 1.293 & 0.422 & 1.395 & 2.239 & 0.844 \\
\hline $\mathrm{CrO}_{2} / \mathrm{Ir}$ & 0.856 & 1.266 & 0.410 & 1.389 & 2.236 & 0.848 \\
\hline $\begin{array}{c}\mathrm{CrO}_{2} / \\
\mathrm{Mn}\end{array}$ & 0.819 & 1.250 & 0.431 & 1.456 & 2.339 & 0.883 \\
\hline $\begin{array}{c}\mathrm{CrO}_{2} / \\
\mathrm{Mo}\end{array}$ & 0.904 & 1.302 & 0.398 & 1.318 & 2.163 & 0.845 \\
\hline $\begin{array}{c}\mathrm{CrO}_{2} / \\
\mathrm{Os}\end{array}$ & 0.861 & 1.267 & 0.406 & 1.375 & 2.223 & 0.848 \\
\hline $\begin{array}{c}\mathrm{CrO}_{2} / \\
\mathrm{Ti}\end{array}$ & 0.806 & 1.181 & 0.375 & 1.318 & 2.506 & 1.188 \\
\hline $\mathrm{CrO}_{2} / \mathrm{V}$ & 0.878 & 1.304 & 0.426 & 1.369 & 2.261 & 0.892 \\
\hline $\begin{array}{c}\mathrm{GeO}_{2} / \\
\mathrm{Ir}\end{array}$ & 0.821 & 1.244 & 0.423 & 1.447 & 2.319 & 0.872 \\
\hline $\begin{array}{c}\mathrm{GeO}_{2} / \\
\mathrm{Ti}\end{array}$ & 0.781 & 1.169 & 0.389 & 1.392 & 2.573 & 1.181 \\
\hline $\begin{array}{c}\mathrm{GeO}_{2} / \\
\mathrm{V}\end{array}$ & 0.757 & 1.151 & 0.394 & 1.433 & 2.602 & 1.169 \\
\hline $\mathrm{IrO}_{2} / \mathrm{Cr}$ & 0.802 & 1.225 & 0.422 & 1.486 & 2.360 & 0.874 \\
\hline $\begin{array}{c}\mathrm{IrO}_{2} / \\
\mathrm{Ge}\end{array}$ & 1.039 & 1.250 & 0.419 & 1.284 & 1.679 & 0.833 \\
\hline $\begin{array}{c}\mathrm{IrO}_{2} / \\
\mathrm{Mn}\end{array}$ & 0.796 & 1.226 & 0.429 & 1.503 & 2.384 & 0.881 \\
\hline $\mathrm{IrO}_{2} / \mathrm{Os}$ & 1.039 & 1.239 & 0.396 & 1.250 & 1.652 & 0.817 \\
\hline $\begin{array}{c}\mathrm{IrO}_{2} / \\
\mathrm{Ru}\end{array}$ & 1.035 & 1.238 & 0.402 & 1.262 & 1.666 & 0.832 \\
\hline $\mathrm{IrO}_{2} / \mathrm{Ti}$ & 1.019 & 1.186 & 0.375 & 1.241 & 1.591 & 0.801 \\
\hline $\mathrm{IrO}_{2} / \mathrm{V}$ & 1.029 & 1.219 & 0.396 & 1.261 & 1.643 & 0.824 \\
\hline $\begin{array}{c}\mathrm{MnO}_{2} / \\
\mathrm{Cr}\end{array}$ & 0.815 & 1.239 & 0.425 & 1.452 & 2.340 & 0.888 \\
\hline
\end{tabular}




\begin{tabular}{|c|c|c|c|c|c|c|}
\hline $\begin{array}{c}\mathrm{MnO}_{2} / \\
\mathrm{Ge}\end{array}$ & 0.934 & 1.366 & 0.432 & 1.349 & 2.195 & 0.846 \\
\hline $\begin{array}{c}\mathrm{MnO}_{2} / \\
\mathrm{Mo}\end{array}$ & 0.877 & 1.286 & 0.408 & 1.362 & 2.231 & 0.869 \\
\hline $\begin{array}{c}\mathrm{MnO}_{2} / \\
\mathrm{Ti}\end{array}$ & 0.788 & 1.174 & 0.386 & 1.366 & 2.535 & 1.169 \\
\hline $\begin{array}{c}\mathrm{MoO}_{2} / \\
\mathrm{Ge}\end{array}$ & 0.754 & 1.173 & 0.419 & 1.646 & 2.481 & 0.835 \\
\hline $\begin{array}{c}\mathrm{MoO}_{2} / \\
\mathrm{Ir}\end{array}$ & 0.755 & 1.158 & 0.404 & 1.632 & 2.465 & 0.834 \\
\hline $\begin{array}{c}\mathrm{MoO}_{2} / \\
\mathrm{Mn}\end{array}$ & 0.729 & 1.154 & 0.425 & 1.622 & 2.480 & 0.858 \\
\hline $\begin{array}{c}\mathrm{MoO}_{2} / \\
\mathrm{Os}\end{array}$ & 0.767 & 1.161 & 0.396 & 1.612 & 2.432 & 0.826 \\
\hline $\begin{array}{c}\mathrm{MoO}_{2} / \\
\mathrm{Rh}\end{array}$ & 0.739 & 1.153 & 0.414 & 1.662 & 2.513 & 0.851 \\
\hline $\begin{array}{c}\mathrm{MoO}_{2} / \\
\mathrm{Ru}\end{array}$ & 0.786 & 1.188 & 0.404 & 1.635 & 2.463 & 0.840 \\
\hline $\begin{array}{c}\mathrm{MoO}_{2} / \\
\mathrm{Ti}\end{array}$ & 0.808 & 1.209 & 0.401 & 1.533 & 2.376 & 0.843 \\
\hline $\begin{array}{c}\mathrm{MoO}_{2} / \\
\mathrm{V}\end{array}$ & 0.734 & 1.150 & 0.416 & 1.601 & 2.465 & 0.865 \\
\hline $\begin{array}{c}\mathrm{OsO}_{2} / \\
\mathrm{Cr}\end{array}$ & 0.828 & 1.244 & 0.416 & 1.446 & 2.302 & 0.856 \\
\hline $\mathrm{OsO}_{2} / \mathrm{Ir}$ & 0.850 & 1.254 & 0.405 & 1.406 & 2.239 & 0.833 \\
\hline $\begin{array}{c}\mathrm{OsO}_{2} / \\
\mathrm{Mn}\end{array}$ & 0.815 & 1.237 & 0.422 & 1.468 & 2.326 & 0.858 \\
\hline $\begin{array}{c}\mathrm{OsO}_{2} / \\
\mathrm{Mo}\end{array}$ & 0.869 & 1.268 & 0.399 & 1.366 & 2.224 & 0.858 \\
\hline $\begin{array}{c}\mathrm{OsO}_{2} / \\
\mathrm{Ru}\end{array}$ & 0.851 & 1.258 & 0.407 & 1.404 & 2.251 & 0.846 \\
\hline $\begin{array}{c}\mathrm{OsO}_{2} / \\
\mathrm{Ti}\end{array}$ & 0.947 & 1.401 & 0.454 & 1.345 & 2.350 & 1.005 \\
\hline $\begin{array}{c}\mathrm{OsO}_{2} / \\
\mathrm{V}\end{array}$ & 0.843 & 1.254 & 0.411 & 1.414 & 2.275 & 0.861 \\
\hline $\begin{array}{c}\mathrm{RhO}_{2} / \\
\mathrm{Cr}\end{array}$ & 0.781 & 1.205 & 0.424 & 1.505 & 2.414 & 0.909 \\
\hline $\begin{array}{c}\mathrm{RhO}_{2} / \\
\mathrm{Ge}\end{array}$ & 0.819 & 1.247 & 0.428 & 1.475 & 2.336 & 0.861 \\
\hline
\end{tabular}




\begin{tabular}{|c|c|c|c|c|c|c|}
\hline $\begin{array}{c}\mathrm{RhO}_{2} / \\
\mathrm{Ir}\end{array}$ & 0.819 & 1.228 & 0.409 & 1.444 & 2.297 & 0.853 \\
\hline $\begin{array}{c}\mathrm{RhO}_{2} / \\
\mathrm{Mn}\end{array}$ & 0.793 & 1.222 & 0.429 & 1.502 & 2.394 & 0.892 \\
\hline $\begin{array}{c}\mathrm{RhO}_{2} / \\
\mathrm{Mo}\end{array}$ & 0.860 & 1.267 & 0.407 & 1.388 & 2.261 & 0.873 \\
\hline $\begin{array}{c}\mathrm{RhO}_{2} / \\
\mathrm{Ru}\end{array}$ & 0.825 & 1.235 & 0.410 & 1.435 & 2.301 & 0.866 \\
\hline $\begin{array}{c}\mathrm{RhO}_{2} / \\
\mathrm{Ti}\end{array}$ & 0.780 & 1.171 & 0.391 & 1.401 & 2.559 & 1.158 \\
\hline $\begin{array}{c}\mathrm{RhO}_{2} / \\
\mathrm{V}\end{array}$ & 0.765 & 1.164 & 0.399 & 1.451 & 2.530 & 1.079 \\
\hline $\begin{array}{c}\mathrm{RuO}_{2} / \\
\mathrm{Cr}\end{array}$ & 0.806 & 1.224 & 0.418 & 1.470 & 2.347 & 0.877 \\
\hline $\begin{array}{c}\mathrm{RuO}_{2} / \\
\mathrm{Ge}\end{array}$ & 0.832 & 1.256 & 0.424 & 1.456 & 2.305 & 0.850 \\
\hline $\begin{array}{c}\mathrm{RuO}_{2} / \\
\mathrm{Ir}\end{array}$ & 0.848 & 1.253 & 0.405 & 1.407 & 2.245 & 0.839 \\
\hline $\begin{array}{c}\mathrm{RuO}_{2} / \\
\mathrm{Mn}\end{array}$ & 0.809 & 1.233 & 0.424 & 1.477 & 2.344 & 0.867 \\
\hline $\begin{array}{c}\mathrm{RuO}_{2} / \\
\mathrm{Mo}\end{array}$ & 0.866 & 1.270 & 0.404 & 1.373 & 2.250 & 0.877 \\
\hline $\begin{array}{c}\mathrm{RuO}_{2} / \\
\mathrm{Os}\end{array}$ & 0.851 & 1.252 & 0.401 & 1.395 & 2.234 & 0.839 \\
\hline $\begin{array}{c}\mathrm{RuO}_{2} / \\
\mathrm{Rh}\end{array}$ & 0.832 & 1.247 & 0.415 & 1.437 & 2.295 & 0.858 \\
\hline $\begin{array}{c}\mathrm{RuO}_{2} / \\
\mathrm{Ti}\end{array}$ & 0.786 & 1.173 & 0.387 & 1.389 & 2.535 & 1.145 \\
\hline $\begin{array}{c}\mathrm{RuO}_{2} / \\
\mathrm{V}\end{array}$ & 0.888 & 1.342 & 0.453 & 1.392 & 2.395 & 1.002 \\
\hline $\begin{array}{c}\mathrm{TiO}_{2} / \\
\mathrm{Cr}\end{array}$ & 0.750 & 1.146 & 0.396 & 1.488 & 2.619 & 1.131 \\
\hline $\begin{array}{c}\mathrm{TiO}_{2} / \\
\mathrm{Ge}\end{array}$ & 0.772 & 1.162 & 0.390 & 1.453 & 2.529 & 1.076 \\
\hline $\mathrm{TiO}_{2} / \mathrm{Ir}$ & 0.817 & 1.234 & 0.418 & 1.485 & 2.355 & 0.871 \\
\hline $\begin{array}{c}\mathrm{TiO}_{2} / \\
\mathrm{Mn}\end{array}$ & 0.749 & 1.163 & 0.415 & 1.530 & 2.615 & 1.085 \\
\hline $\begin{array}{c}\mathrm{TiO}_{2} / \\
\mathrm{Mo}\end{array}$ & 0.853 & 1.266 & 0.413 & 1.426 & 2.308 & 0.882 \\
\hline
\end{tabular}




\begin{tabular}{|c|c|c|c|c|c|c|}
\hline $\begin{array}{c}\mathrm{TiO}_{2} / \\
\mathrm{Rh}\end{array}$ & 0.813 & 1.239 & 0.426 & 1.497 & 2.388 & 0.890 \\
\hline $\begin{array}{c}\mathrm{TiO}_{2} / \\
\mathrm{Ru}\end{array}$ & 0.835 & 1.252 & 0.417 & 1.460 & 2.335 & 0.875 \\
\hline $\mathrm{TiO}_{2} / \mathrm{V}$ & 0.885 & 1.329 & 0.444 & 1.392 & 2.349 & 0.957 \\
\hline $\mathrm{VO}_{2} / \mathrm{Cr}$ & 0.825 & 1.245 & 0.420 & 1.467 & 2.330 & 0.863 \\
\hline $\begin{array}{c}\mathrm{VO}_{2} / \\
\mathrm{Ge}\end{array}$ & 0.969 & 1.396 & 0.426 & 1.328 & 2.161 & 0.832 \\
\hline $\begin{array}{c}\mathrm{VO}_{2} / \\
\mathrm{Mn}\end{array}$ & 0.824 & 1.252 & 0.428 & 1.479 & 2.348 & 0.869 \\
\hline $\begin{array}{c}\mathrm{VO}_{2} / \\
\mathrm{Mo}\end{array}$ & 0.842 & 1.240 & 0.398 & 1.406 & 2.272 & 0.866 \\
\hline $\begin{array}{c}\mathrm{VO}_{2} / \\
\mathrm{Os}\end{array}$ & 0.813 & 1.213 & 0.402 & 1.461 & 2.294 & 0.836 \\
\hline $\begin{array}{c}\mathrm{VO}_{2} / \\
\mathrm{Ru}\end{array}$ & 0.813 & 1.222 & 0.410 & 1.469 & 2.318 & 0.850 \\
\hline $\mathrm{VO}_{2} / \mathrm{Ti}$ & 0.878 & 1.288 & 0.410 & 1.386 & 2.241 & 0.855 \\
\hline $\mathrm{CrO}_{2}$ & 0.834 & 1.253 & 0.419 & 1.421 & 2.286 & 0.866 \\
\hline $\mathrm{GeO}_{2}$ & 0.754 & 1.166 & 0.411 & 1.472 & 2.604 & 1.132 \\
\hline $\mathrm{IrO}_{2}$ & 1.016 & 1.227 & 0.402 & 1.277 & 1.688 & 0.827 \\
\hline $\mathrm{MnO}_{2}$ & 0.821 & 1.254 & 0.433 & 1.460 & 2.351 & 0.892 \\
\hline $\mathrm{MoO}_{2}$ & 0.770 & 1.173 & 0.403 & 1.576 & 2.439 & 0.864 \\
\hline $\mathrm{OsO}_{2}$ & 0.852 & 1.255 & 0.403 & 1.398 & 2.234 & 0.836 \\
\hline $\mathrm{RhO}_{2}$ & 0.802 & 1.221 & 0.419 & 1.476 & 2.350 & 0.874 \\
\hline $\mathrm{RuO}_{2}$ & 0.836 & 1.246 & 0.410 & 1.423 & 2.285 & 0.861 \\
\hline $\mathrm{TiO}_{2}$ & 0.774 & 1.154 & 0.379 & 1.415 & 2.594 & 1.178 \\
\hline $\mathrm{VO}_{2}$ & 0.823 & 1.239 & 0.417 & 1.459 & 2.321 & 0.862 \\
\hline
\end{tabular}

Table S2 Input Energetic and Geometrical Descriptors (5)

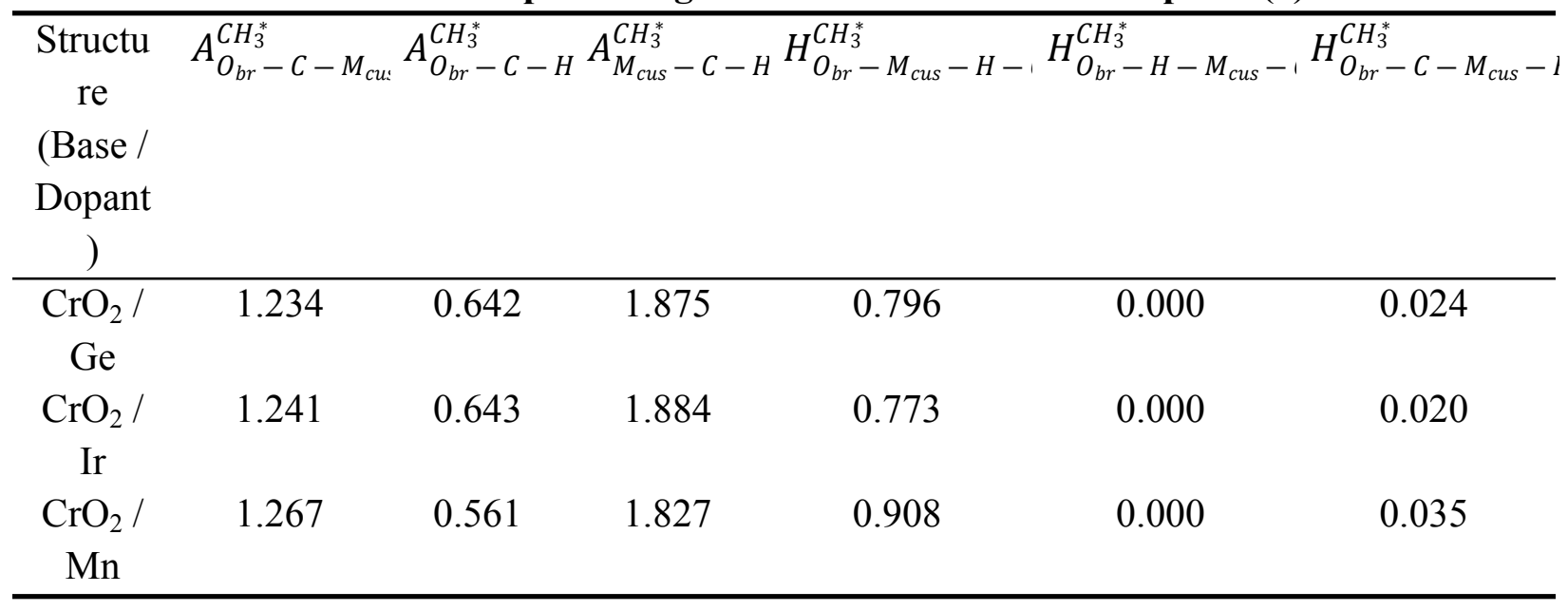




\begin{tabular}{|c|c|c|c|c|c|c|}
\hline $\begin{array}{c}\mathrm{CrO}_{2} / \\
\mathrm{Mo}\end{array}$ & 1.192 & 0.706 & 1.898 & 0.763 & 0.000 & 0.000 \\
\hline $\begin{array}{c}\mathrm{CrO}_{2} / \\
\mathrm{Os}\end{array}$ & 1.234 & 0.654 & 1.888 & 0.767 & 0.000 & 0.020 \\
\hline $\begin{array}{c}\mathrm{CrO}_{2} / \\
\mathrm{Ti}\end{array}$ & 1.131 & 0.448 & 1.578 & 1.126 & 0.000 & 0.014 \\
\hline $\begin{array}{c}\mathrm{CrO}_{2} / \\
\mathrm{V}\end{array}$ & 1.199 & 0.625 & 1.824 & 0.811 & 0.000 & 0.014 \\
\hline $\begin{array}{c}\mathrm{GeO}_{2} / \\
\mathrm{Ir}\end{array}$ & 1.270 & 0.577 & 1.847 & 0.868 & 0.000 & 0.032 \\
\hline $\begin{array}{c}\mathrm{GeO}_{2} / \\
\mathrm{Ti}\end{array}$ & 1.175 & 0.396 & 1.571 & 1.175 & 0.396 & 0.000 \\
\hline $\begin{array}{c}\mathrm{GeO}_{2} / \\
\mathrm{V}\end{array}$ & 1.206 & 0.373 & 1.579 & 1.200 & 0.367 & 0.040 \\
\hline $\begin{array}{c}\mathrm{IrO}_{2} / \\
\mathrm{Cr}\end{array}$ & 1.298 & 0.547 & 1.846 & 0.857 & 0.000 & 0.035 \\
\hline $\begin{array}{c}\mathrm{IrO}_{2} / \\
\mathrm{Ge}\end{array}$ & 1.286 & 1.129 & 1.889 & 0.313 & 0.381 & 0.037 \\
\hline $\begin{array}{c}\mathrm{IrO}_{2} / \\
\mathrm{Mn}\end{array}$ & 1.303 & 0.529 & 1.832 & 0.888 & 0.000 & 0.040 \\
\hline $\begin{array}{c}\mathrm{IrO}_{2} / \\
\mathrm{Os}\end{array}$ & 1.271 & 1.155 & 1.929 & 0.532 & 0.000 & 0.024 \\
\hline $\begin{array}{c}\mathrm{IrO}_{2} / \\
\mathrm{Ru}\end{array}$ & 1.276 & 1.142 & 1.907 & 0.541 & 0.000 & 0.020 \\
\hline $\begin{array}{c}\mathrm{IrO}_{2} / \\
\mathrm{Ti}\end{array}$ & 1.298 & 1.208 & 1.966 & 0.320 & 0.392 & 0.072 \\
\hline $\begin{array}{c}\mathrm{IrO}_{2} / \\
\mathrm{V}\end{array}$ & 1.287 & 1.162 & 1.921 & 0.318 & 0.389 & 0.058 \\
\hline $\begin{array}{c}\mathrm{MnO}_{2} / \\
\mathrm{Cr}\end{array}$ & 1.268 & 0.561 & 1.829 & 0.903 & 0.000 & 0.032 \\
\hline $\begin{array}{c}\mathrm{MnO}_{2} / \\
\mathrm{Ge}\end{array}$ & 1.180 & 0.684 & 1.864 & 0.801 & 0.000 & 0.014 \\
\hline $\begin{array}{c}\mathrm{MnO}_{2} / \\
\mathrm{Mo}\end{array}$ & 1.213 & 0.651 & 1.864 & 0.823 & 0.000 & 0.014 \\
\hline $\begin{array}{c}\mathrm{MnO}_{2} / \\
\mathrm{Ti}\end{array}$ & 1.164 & 0.424 & 1.587 & 1.152 & 0.000 & 0.000 \\
\hline $\begin{array}{c}\mathrm{MoO}_{2} / \\
\mathrm{Ge}\end{array}$ & 1.418 & 0.468 & 1.887 & 1.171 & 0.246 & 0.345 \\
\hline $\begin{array}{c}\mathrm{MoO}_{2} / \\
\mathrm{Ir}\end{array}$ & 1.422 & 0.483 & 1.904 & 1.163 & 0.246 & 0.329 \\
\hline
\end{tabular}




\begin{tabular}{|c|c|c|c|c|c|c|}
\hline $\begin{array}{c}\mathrm{MoO}_{2} / \\
\mathrm{Mn}\end{array}$ & 1.401 & 0.457 & 1.858 & 0.817 & 0.014 & 0.053 \\
\hline $\begin{array}{c}\mathrm{MoO}_{2} / \\
\mathrm{Os}\end{array}$ & 1.416 & 0.509 & 1.919 & 1.143 & 0.256 & 0.308 \\
\hline $\begin{array}{c}\mathrm{MoO}_{2} / \\
\mathrm{Rh}\end{array}$ & 1.431 & 0.445 & 1.877 & 1.190 & 0.230 & 0.356 \\
\hline $\begin{array}{c}\mathrm{MoO}_{2} / \\
\mathrm{Ru}\end{array}$ & 1.415 & 0.494 & 1.898 & 1.145 & 0.246 & 0.311 \\
\hline $\begin{array}{c}\mathrm{MoO}_{2} / \\
\mathrm{Ti}\end{array}$ & 1.348 & 0.550 & 1.898 & 1.106 & 0.317 & 0.230 \\
\hline $\begin{array}{c}\mathrm{MoO}_{2} / \\
\mathrm{V}\end{array}$ & 1.392 & 0.469 & 1.861 & 1.166 & 0.269 & 0.314 \\
\hline $\begin{array}{c}\mathrm{OsO}_{2} / \\
\mathrm{Cr}\end{array}$ & 1.277 & 0.592 & 1.869 & 0.807 & 0.000 & 0.028 \\
\hline $\begin{array}{c}\mathrm{OsO}_{2} / \\
\mathrm{Ir}\end{array}$ & 1.261 & 0.643 & 1.904 & 1.032 & 0.408 & 0.126 \\
\hline $\begin{array}{c}\mathrm{OsO}_{2} / \\
\mathrm{Mn}\end{array}$ & 1.290 & 0.572 & 1.862 & 0.822 & 0.000 & 0.035 \\
\hline $\begin{array}{c}\mathrm{OsO}_{2} / \\
\mathrm{Mo}\end{array}$ & 1.227 & 0.657 & 1.884 & 1.016 & 0.434 & 0.074 \\
\hline $\begin{array}{c}\mathrm{OsO}_{2} / \\
\mathrm{Ru}\end{array}$ & 1.255 & 0.634 & 1.888 & 1.036 & 0.410 & 0.120 \\
\hline $\begin{array}{c}\mathrm{OsO}_{2} / \\
\mathrm{Ti}\end{array}$ & 1.113 & 0.570 & 1.683 & 1.035 & 0.488 & 0.000 \\
\hline $\begin{array}{c}\mathrm{OsO}_{2} / \\
\mathrm{V}\end{array}$ & 1.255 & 0.614 & 1.869 & 1.048 & 0.404 & 0.127 \\
\hline $\begin{array}{c}\mathrm{RhO}_{2} / \\
\mathrm{Cr}\end{array}$ & 1.303 & 0.506 & 1.809 & 0.929 & 0.000 & 0.037 \\
\hline $\begin{array}{c}\mathrm{RhO}_{2} / \\
\mathrm{Ge}\end{array}$ & 1.287 & 0.566 & 1.853 & 1.084 & 0.369 & 0.192 \\
\hline $\begin{array}{c}\mathrm{RhO}_{2} / \\
\mathrm{Ir}\end{array}$ & 1.285 & 0.596 & 1.880 & 1.067 & 0.380 & 0.163 \\
\hline $\begin{array}{c}\mathrm{RhO}_{2} / \\
\mathrm{Mn}\end{array}$ & 1.300 & 0.521 & 1.820 & 0.910 & 0.000 & 0.037 \\
\hline $\begin{array}{c}\mathrm{RhO}_{2} / \\
\mathrm{Mo}\end{array}$ & 1.234 & 0.628 & 1.862 & 1.034 & 0.422 & 0.093 \\
\hline $\begin{array}{c}\mathrm{RhO}_{2} / \\
\mathrm{Ru}\end{array}$ & 1.272 & 0.594 & 1.866 & 1.066 & 0.387 & 0.148 \\
\hline $\begin{array}{c}\mathrm{RhO}_{2} / \\
\mathrm{Ti}\end{array}$ & 1.187 & 0.407 & 1.593 & 1.171 & 0.391 & 0.000 \\
\hline
\end{tabular}




\begin{tabular}{|c|c|c|c|c|c|c|}
\hline $\begin{array}{c}\mathrm{RhO}_{2} / \\
\mathrm{V}\end{array}$ & 1.238 & 0.425 & 1.664 & 1.172 & 0.360 & 0.093 \\
\hline $\begin{array}{c}\mathrm{RuO}_{2} / \\
\mathrm{Cr}\end{array}$ & 1.290 & 0.557 & 1.847 & 0.851 & 0.000 & 0.032 \\
\hline $\begin{array}{c}\mathrm{RuO}_{2} / \\
\mathrm{Ge}\end{array}$ & 1.278 & 0.590 & 1.868 & 1.066 & 0.382 & 0.174 \\
\hline $\begin{array}{c}\mathrm{RuO}_{2} / \\
\mathrm{Ir}\end{array}$ & 1.261 & 0.638 & 1.898 & 1.035 & 0.407 & 0.125 \\
\hline $\begin{array}{c}\mathrm{RuO}_{2} / \\
\mathrm{Mn}\end{array}$ & 1.292 & 0.559 & 1.851 & 0.844 & 0.000 & 0.035 \\
\hline $\begin{array}{c}\mathrm{RuO}_{2} / \\
\mathrm{Mo}\end{array}$ & 1.223 & 0.637 & 1.860 & 1.027 & 0.431 & 0.076 \\
\hline $\begin{array}{c}\mathrm{RuO}_{2} / \\
\mathrm{Os}\end{array}$ & 1.255 & 0.647 & 1.902 & 1.029 & 0.413 & 0.112 \\
\hline $\begin{array}{c}\mathrm{RuO}_{2} / \\
\mathrm{Rh}\end{array}$ & 1.271 & 0.598 & 1.869 & 1.061 & 0.389 & 0.153 \\
\hline $\begin{array}{c}\mathrm{RuO}_{2} / \\
\mathrm{Ti}\end{array}$ & 1.184 & 0.425 & 1.609 & 1.158 & 0.398 & 0.000 \\
\hline $\begin{array}{c}\mathrm{RuO}_{2} / \\
\mathrm{V}\end{array}$ & 1.158 & 0.528 & 1.686 & 1.076 & 0.446 & 0.055 \\
\hline $\begin{array}{c}\mathrm{TiO}_{2} / \\
\mathrm{Cr}\end{array}$ & 1.251 & 0.364 & 1.614 & 1.214 & 0.000 & 0.020 \\
\hline $\begin{array}{c}\mathrm{TiO}_{2} / \\
\mathrm{Ge}\end{array}$ & 1.245 & 0.430 & 1.675 & 1.171 & 0.356 & 0.089 \\
\hline $\begin{array}{c}\mathrm{TiO}_{2} / \\
\mathrm{Ir}\end{array}$ & 1.297 & 0.556 & 1.853 & 1.093 & 0.358 & 0.191 \\
\hline $\begin{array}{c}\mathrm{TiO}_{2} / \\
\mathrm{Mn}\end{array}$ & 1.277 & 0.365 & 1.642 & 1.208 & 0.000 & 0.028 \\
\hline $\begin{array}{c}\mathrm{TiO}_{2} / \\
\mathrm{Mo}\end{array}$ & 1.251 & 0.596 & 1.847 & 1.057 & 0.400 & 0.123 \\
\hline $\begin{array}{c}\mathrm{TiO}_{2} / \\
\mathrm{Rh}\end{array}$ & 1.294 & 0.532 & 1.826 & 1.107 & 0.353 & 0.198 \\
\hline $\begin{array}{c}\mathrm{TiO}_{2} / \\
\mathrm{Ru}\end{array}$ & 1.276 & 0.573 & 1.849 & 1.077 & 0.377 & 0.162 \\
\hline $\begin{array}{c}\mathrm{TiO}_{2} / \\
\mathrm{V}\end{array}$ & 1.178 & 0.562 & 1.740 & 1.059 & 0.442 & 0.069 \\
\hline $\begin{array}{c}\mathrm{VO}_{2} / \\
\mathrm{Cr}\end{array}$ & 1.285 & 0.574 & 1.858 & 0.833 & 0.000 & 0.032 \\
\hline $\begin{array}{c}\mathrm{VO}_{2} / \\
\mathrm{Ge}\end{array}$ & 1.164 & 0.719 & 1.883 & 0.952 & 0.491 & 0.024 \\
\hline
\end{tabular}




\begin{tabular}{ccccccc}
\hline $\begin{array}{c}\mathrm{VO}_{2} / \\
\mathrm{Mn}\end{array}$ & 1.285 & 0.559 & 1.844 & 0.861 & 0.000 & 0.035 \\
$\mathrm{VO}_{2} /$ & 1.257 & 0.620 & 1.878 & 1.047 & 0.403 & 0.112 \\
$\mathrm{Mo}$ & & & & & & \\
$\mathrm{VO}_{2} /$ & 1.307 & 0.600 & 1.904 & 1.069 & 0.364 & 0.181 \\
$\mathrm{Os}$ & & & & & & \\
$\mathrm{VO}_{2} /$ & 1.301 & 0.582 & 1.882 & 1.078 & 0.363 & 0.185 \\
$\mathrm{Ru}$ & & & & & & \\
$\mathrm{VO}_{2} /$ & 1.230 & 0.646 & 1.876 & 1.021 & 0.428 & 0.092 \\
$\mathrm{Ti}$ & & & & & & \\
$\mathrm{CrO}_{2}$ & 1.255 & 0.602 & 1.856 & 0.854 & 0.000 & 0.028 \\
$\mathrm{GeO}_{2}$ & 1.229 & 0.369 & 1.598 & 1.207 & 0.000 & 0.020 \\
$\mathrm{IrO}_{2}$ & 1.286 & 1.118 & 1.913 & 0.555 & 0.014 & 0.014 \\
$\mathrm{MnO}_{2}$ & 1.264 & 0.553 & 1.817 & 0.914 & 0.000 & 0.035 \\
$\mathrm{MoO}_{2}$ & 1.376 & 0.500 & 1.874 & 0.778 & 0.000 & 0.040 \\
$\mathrm{OsO}_{2}$ & 1.256 & 0.646 & 1.903 & 0.740 & 0.000 & 0.020 \\
$\mathrm{RhO}_{2}$ & 1.294 & 0.554 & 1.849 & 0.864 & 0.000 & 0.035 \\
$\mathrm{RuO}_{2}$ & 1.264 & 0.607 & 1.870 & 0.809 & 0.000 & 0.024 \\
$\mathrm{TiO}_{2}$ & 1.199 & 0.385 & 1.584 & 1.189 & 0.000 & 0.000 \\
$\mathrm{VO}_{2}$ & 1.284 & 0.579 & 1.863 & 0.841 & 0.014 & 0.032 \\
\hline & & & & & &
\end{tabular}




\section{Concepts of LASSO and Percentile-LASSO}

LASSO is an advanced linear regression method, which is also known as the $L_{1}$ penalized regression in the field of optimization. ${ }^{\mathrm{S} 1} \mathrm{~A}$ major characteristic of LASSO is to shrink the number of features with nonzero coefficients with a tuning hyperparameter which is usually denoted by $\lambda$ in mathematical textbooks while $\alpha$ in computer programs. For consistency, $\alpha$ denotes this tuning hyperparameter both in the main text and this supporting information. In some literatures, $\alpha$ is also called the penalized parameter.

For illustrative purposes, it is assumed that there has a sample of $n$ observations and for each observation there is a target $y$ and a feature vector $\left(x_{1}, \ldots, x_{p}\right)$ if $p$ features were given. Thus, the optimization objective $\epsilon$ for LASSO can be constructed as eq S1, where $\beta_{0}$ is the intercept and $\beta_{1}$ to $\beta_{p}$ are coefficients of features.

$$
\epsilon=\underset{\beta}{\arg \min }\left\{\sum_{i=1}^{n}\left(y_{i}-\beta_{0}-\sum_{j=1}^{p} x_{i j} \beta_{j}\right)^{2}+\alpha \sum_{j=1}^{p}\left|\beta_{j}\right|\right\}
$$

The major difference between ordinary linear regression and LASSO is that only few of features in LASSO have nonzero coefficients which reflect feature importance. The degree of the shrinkage of features is controlled by the penalized parameter $\alpha$. In practice, a value for $\alpha$ is chosen with the aid of cross-validation. ${ }^{\mathrm{S} 2}$ However, it is shown that LASSO is extremely sensitive to the fold assignment. LASSO using different $\alpha$ selected by random fold splits is prone to give unstable results (especially fluctuations in the number of features with nonzero coefficients), which will leave further analyses difficult to undergo. To tackle this model-selection instability issue, Percentile-LASSO is proposed with the introduce of another hyperparameter $\theta .{ }^{\mathrm{S} 3}$

Before delving into details of Percentile-LASSO, Figure S1 gives a motivating example. ${ }^{\mathrm{S} 4} \mathrm{With}$ a number of different training and test fold assignments, the best $\alpha$ for LASSO chosen based on a specific fold assignment can be extremely different, which consequently gives various number of features with nonzero coefficients. It is necessary that hyperparameters selected by crossvalidation should not largely affect the final results. Fortunately, the tendency of $\alpha$ chosen from different fold assignments versus respective number of nonzero coefficients behaves like a decreasing curve, i.e. the greater $\alpha$ is, the fewer nonzero features extract. However, larger $\alpha$ does not mean smaller cross-validation error. In practical terms, $\alpha$ at various percentiles are used to train LASSO and the $\alpha$ with the minimum cross-validation error is the best. The value of each percentile is so-called percentile parameter $\theta$. 


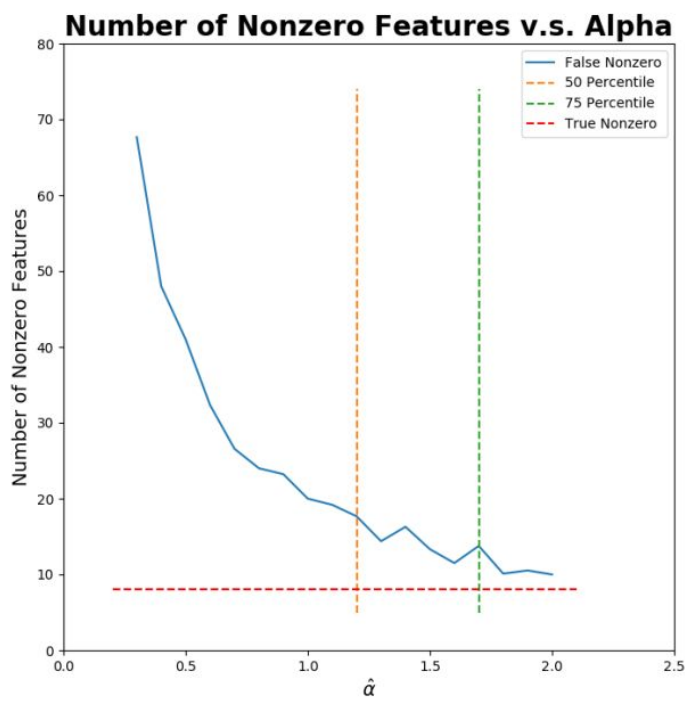

Figure S1. This figure is for illustrative purposes. Training results from real world data can be found in the reference S4. The blue curve (False Nonzero) denotes the number of nonzero features given by LASSO with specific $\alpha$. The red curve (True Nonzero) denotes the number of nonzero features the linear relation actually has. The orange and green curve denote the value of $\alpha$ at 50 and 75 percentile respectively. With greater penalized $\alpha$, LASSO is prone to extract fewer nonzero features.

Percentile-LASSO is a straightforward modification of standard LASSO discussed before. With the aid of percentile parameter $\theta$, the results given by LASSO will be more stable. Table S3 gives the pseudocode of the algorithm of percentile-LASSO. 
Table S3 Pseudocode of Percentile-LASSO

Pseudocode of Percentile-LASSO

1 Set $A=\left(\alpha_{1}, \ldots, \alpha_{n}\right)$ as a sequence of penalized parameter $\alpha$ values.

2 for $m=1$ to $M$ do

Randomly split dataset into $\mathrm{k}$ folds ( $\mathrm{k}-1$ folds are the training set, 1 fold is the test set).

for $\alpha=\alpha_{1}$ to $\alpha_{n}$ do

Train the standard LASSO on k different training sets and calculate respective MSE.

\section{end}

Let $\widehat{\alpha_{m}}$ be the $\alpha$ with which standard LASSO has the minimum average MSE.

\section{end}

3 Let $\hat{\mathrm{A}}=\left(\widehat{\alpha_{1}}, \ldots, \hat{\alpha_{m}}\right)$ denotes a sequence of estimated best $\alpha$ from $\mathrm{M}$ times LASSO trainings.

4 Set $\Theta=\left(\theta_{1}, \ldots, \theta_{r}\right)$ as a sequence of percentile parameter $\theta$ values.

5 for $\theta=\theta_{1}$ to $\theta_{r}$ do

Compute $\hat{\alpha}(\theta)$ according to the $\theta$-percentile of $\hat{\mathrm{A}}$.

Retraining standard LASSO with $\hat{\alpha}(\theta)$ by k-fold cross-validation (similar to the one in step 2).

end

6 Select $\hat{\theta}$ be the value of $\theta$ in $\Theta$ with the minimum average MSE.

7 The solution of percentile-LASSO is the solution of standard LASSO trained with $\alpha=\hat{\alpha}$ ( $\hat{\theta})$.

The series $A$ and $\Theta$ which determine $\alpha$ and $\theta$ respectively are set according to specific dataset. $\alpha$ used in this study ranges from 0.001 to 0.5 at an interval of $0.001 . \alpha$ larger than 0.2 is not chosen by the cross-validation, which shows that the range of $\alpha$ above is quite reasonable. On the other hand, smaller $\theta$ means smaller $\alpha$ which gives out much more nonzero features possibly including more noises. Thus, small $\theta$ is not recommendable and the sequence of $\theta$ from 50 to 100 at an interval of 5 is a fair estimation. The study in the main text also shows that even with a relative large $\alpha$ determined by a large $\theta$ the final nonzero features can still include insignificant ones due to the numerical instability, which is amended by a second feature selection. 


\section{Comparasion of Different Regression Methods}

Various regression methods were evaluated. Linear methods consist of ordinary least-squares regression (OLS), least absolute shrinkage and selection operator (LASSO), ridge regression (RR). Nonlinear methods are made up of support vector machines regression (SVR) ${ }^{\mathrm{S} 5}$, and gradient boosting regression $(\mathrm{GBR})^{\mathrm{S} 6}$. Moreover, only can LASSO and GBR accomplish an effective feature selection, which gets rid of insignificant features to carry out further analysis.

The input descriptors (features) include 2 energetic descriptors $\left(E_{H^{*}}\right.$ and $\left.E_{C_{3}^{*}}\right)$ and 28 geometrical descriptors. The hyperparameters of these machine learning methods are adopted with the default values in scikit-learn python package except for GBR where the number of tree estimators is set to 200. Table S4 and Table S5 illustrate the performance of different methods on the dataset of the surface-stabilized mechanism (65 samples) and the radical mechanism (35 samples) respectively.

Table S4 Comparasion of Regression Methods for the Surface-stabilized Mechanism

\begin{tabular}{ccccc}
\hline ML Methods & \multicolumn{2}{c}{ Training Set } & \multicolumn{2}{c}{ Test Set } \\
\cline { 2 - 5 } & $\mathrm{R}^{2}(\mu / \sigma)$ & $\operatorname{MSE}(\mu / \sigma) / \mathrm{eV}$ & $\mathrm{R}^{2}(\mu / \sigma)$ & $\mathrm{MSE}(\mu / \sigma) / \mathrm{eV}$ \\
\hline OLS & $0.96 / 0.00$ & $0.0070 / 0.0000$ & $0.23 / 0.48$ & $0.1230 / 0.0117$ \\
RR & $0.94 / 0.00$ & $0.0116 / 0.0000$ & $0.70 / 0.03$ & $0.0476 / 0.0007$ \\
LASSO & $0.92 / 0.00$ & $0.0150 / 0.0000$ & $0.81 / 0.01$ & $0.0320 / 0.0002$ \\
SVR & $0.86 / 0.00$ & $0.0252 / 0.0000$ & $0.68 / 0.02$ & $0.0563 / 0.0006$ \\
GBR & $1.00 / 0.00$ & $0.0000 / 0.0000$ & $0.68 / 0.01$ & $0.0547 / 0.0002$ \\
& & & \multicolumn{3}{c}{$(\mu$ : Mean $\sigma:$ Variance) } \\
\hline
\end{tabular}

Table S5 Comparasion of Different Regression Methods for the Radical Mechanism

\begin{tabular}{ccccc}
\hline ML Methods & \multicolumn{2}{c}{ Training Set } & \multicolumn{2}{c}{ Test Set } \\
\cline { 2 - 5 } & $\mathrm{R}^{2}(\mu / \sigma)$ & $\operatorname{MSE}(\mu / \sigma) / \mathrm{eV}$ & $\mathrm{R}^{2}(\mu / \sigma)$ & $\operatorname{MSE}(\mu / \sigma) / \mathrm{eV}$ \\
\hline OLS & $1.00 / 0.00$ & $0.0000 / 0.0000$ & - & - \\
RR & $0.97 / 0.00$ & $0.0038 / 0.0000$ & $0.67 / 0.03$ & $0.0452 / 0.0007$ \\
LASSO & $0.96 / 0.00$ & $0.0062 / 0.0000$ & $0.90 / 0.00$ & $0.0140 / 0.0001$ \\
SVR & $0.82 / 0.00$ & $0.0256 / 0.0000$ & $0.41 / 0.04$ & $0.0810 / 0.0007$ \\
GBR & $1.00 / 0.00$ & $0.0000 / 0.0000$ & $0.81 / 0.01$ & $0.0264 / 0.0001$ \\
& & & \multicolumn{2}{c}{$(\mu$ : Mean $\sigma$ : Variance $)$} \\
\hline
\end{tabular}

OLS can fit the training set very well but have a terrible performance on the test set, which even fails to predict the test set of the radical mechanism due to the overfitting. RR is also not satisfying because of its weak variability. For nonlinear methods, GBR has a better performance than SVR both on the training set and the test set. However, the intrinsic characteristic of nonlinear methods make it difficult to interpret the importance of each feature, i.e. physical meanings of descriptors are hard to reveal. Among all of methods compared above, LASSO excels itself to have a fair variability and interpretability. Thus, LASSO is used as the main regression method in this study. 


\section{References}

S1. Tibshirani, R. Regression Shrinkage and Selection via the Lasso. J. R. Statist. Soc. B 1996, 58 (1), 267-288.

S2. Burman, P. A Comparative Study of Ordinary Cross-validation, V-fold Cross-validation and the Repeated Learning-testing Methods. Biometrika 1989, 76 (3), 503-514.

S3. Roberts, S.; Nowak, G. Stabilizing the Lasso against Cross-validation Variability. Computational Statistics and Data Analysis 2014, 70, 198-211.

S4. Wang, X.; Park, T.; Carriere, K. C. Variable Selection via Combined Penalization for Highdimensional Data Analysis, Computer Statistics and Data Analysis, 2010, 54(10), 2230-2243.

S5. Drucker, H.; Burges, C. J. C.; Kaufman, L.; Smola, A. J.; Vapnik, V. Support Vector Regression Machines. Adv. Neural Inf. Process. Syst. 1997, 9, 155-161.

S6. Friedman, J. H. Greedy Function Approximation: A Gradient Boosting Machine. Ann. Stat. 2001, 29, 1189-1232. 